\title{
Anti-terrorism politics and the risk of provoking
}

Citation for published version (APA):

Dietrich, F. K. (2008). Anti-terrorism politics and the risk of provoking. METEOR, Maastricht University School of Business and Economics. METEOR Research Memorandum No. 011 https://doi.org/10.26481/umamet.2008011

Document status and date:

Published: 01/01/2008

DOI:

10.26481/umamet.2008011

Document Version:

Publisher's PDF, also known as Version of record

\section{Please check the document version of this publication:}

- A submitted manuscript is the version of the article upon submission and before peer-review. There can be important differences between the submitted version and the official published version of record.

People interested in the research are advised to contact the author for the final version of the publication, or visit the DOI to the publisher's website.

- The final author version and the galley proof are versions of the publication after peer review.

- The final published version features the final layout of the paper including the volume, issue and page numbers.

Link to publication

\footnotetext{
General rights rights.

- You may freely distribute the URL identifying the publication in the public portal. please follow below link for the End User Agreement:

www.umlib.nl/taverne-license

Take down policy

If you believe that this document breaches copyright please contact us at:

repository@maastrichtuniversity.nl

providing details and we will investigate your claim.
}

Copyright and moral rights for the publications made accessible in the public portal are retained by the authors and/or other copyright owners and it is a condition of accessing publications that users recognise and abide by the legal requirements associated with these

- Users may download and print one copy of any publication from the public portal for the purpose of private study or research.

- You may not further distribute the material or use it for any profit-making activity or commercial gain

If the publication is distributed under the terms of Article $25 \mathrm{fa}$ of the Dutch Copyright Act, indicated by the "Taverne" license above, 
Franz Dietrich

Anti-terrorism politics and the risk of provoking

$\mathrm{RM} / 08 / 011$

JEL code: B4, C7, C9, F5

\section{METE@R}

Maastricht research school of Economics of TEchnology and ORganizations

Universiteit Maastricht

Faculty of Economics and Business Administration P.O. Box 616

NL - 6200 MD Maastricht

phone : :++31433883830

fax $\quad$ : ++31433884873 


\title{
Anti-terrorism politics and the risk of provoking
}

\author{
Franz Dietrich ${ }^{1}$
}

May 2008

\begin{abstract}
A population's level of terrorism depends on two factors: people's preferences (would they like creating damage?) and the constraints under which people act (what damage could they create, and at what punishment?). Causerelated policies, e.g. improving social stability or education, aim at appeasing preferences, thereby reducing terrorism. Symptom-related policies, e.g. embargoes or wars, change the constraints ('deterrence'), but may have side effects on preferences ('provocation'); terrorism increases if provocation overweighs deterrence. I model preferences for damage as endogenous and policy-dependent. I argue that provocation by tough policies is easy to overlook, and show that provocation-neglect leads to toughness-exaggeration.
\end{abstract}

Keywords. Endogenous preferences, reciprocity, dynamic inconsistency, terrorism

\section{Introduction}

Rational-choice-based crime prevention models ${ }^{2}$ do usually not carry over as such to the problem of terrorism prevention; here, a different approach is due, or so I claim. The reason is the phenomenon of provocation, which terrorism politics faces to a larger extent than crime politics. Provocation, as construed in this paper, is the development of a desire to create damage in response to the policy (e.g. to a war). In order to account for provocation, a model must endogenise the (degree of) damage-proneness of individuals. Why is provocation a crucial ingredient when modelling terrorism, more so than when modelling regular criminality? The answer lies in the motivation underlying terrorism. The motivation underlying regular crimes is typically not related to the policy makers but to monetary or other material advantages (for property crimes) or to emotions about certain persons (for hate crimes). By contrast, a terrorist's motivation is often related to (his feelings about) those cultures, governments or countries that are in charge of the anti-terrorism policy or are associated with it. So, the motivation underlying terrorism can be affected (i.e., created, increased, or reduced) by the anti-terrorism policy, whereas the motivation driving regular crimes might be exogenously given. In short, theft is not motivated by hate against those pursuing thieves, but terrorism may be motivated by hate against those pursuing terrorists, and this hate may be provoked by the policy itself

\footnotetext{
${ }^{1}$ Maastricht University \& LSE. Homepage: www.personeel.unimaas.nl/f.dietrich

${ }^{2}$ See Michael Tonry (1998) for an overview of the empirical and theoretical crime literature.
} 
(particularly, by toughness). As a result, policy makers and our models should (i) treat an individual's desire to create terrorism as instable and endogenously responding to the policy, and (ii) focus not only on individuals with currently brutal desires but also on currently peaceful individuals who might be provoked.

This theoretical paper proposes such a model. It shows that the policy maker faces a trade-off between deterrence and provocation, and compares provocation-aware with provocation-neglecting policies. Being not empirical, the paper makes no concrete claims on the size of the provocation effect of contemporary or past anti-terrorism policies, nor on whether these policies resemble more the provocation-aware or the provocation-neglecting policies analysed here. But I argue that provocation is indeed easy to systematically overlook in practice, and I show that provocation-neglect leads to toughness exaggeration. The paper is organised into an informal part (Section 2) that introduces some key ideas, and a formal part (Section 3) that provides the mathematical foundations, among other things giving sufficient conditions under which the marginal effect of toughness is the sum of a terrorism-reducing deterrence effect and a terrorism-increasing provocation effect.

Although provocation by anti-terrorism policies is discussed in political science and sociology (and by people on the streets), it has to my knowledge not been investigated formally in a rational-choice-based model. In focussing on provocation and modelling it as a form of preference/taste change, I take the approach of the economic literature on endogenous preferences. The instability of tastes and their endogenous determination by environmental factors such as governmental policies or institutions is increasingly recognised and modelled in economics (e.g. Robert Polak 1976, Sven Ove Hansson 1995, Gary Becker 1996, Samuel Bowles 1998, Natthew Rabin 1998, Dekel et al. 2007). It is important to incorporate this approach into terrorism modelling, because dispositions towards terrorism seem particularly instable and environment-sensitive in that they typically reflect complex mental states rather than basic biological attributes or needs.

Provocation as modelled here (that is: the emergence of a desire to create damage in response to a policy, e.g. to a war) can be interpreted either as a rational taste change, derived from stable extended preferences over extended alternatives that contain the policy as a taste parameter, or as a dynamically inconsistent taste change. The first interpretation allows one to explain provocation by a stable desire to reciprocate, i.e. to harm tough policy makers (countries, cultures etc.) and to be mild to soft ones. Violent responses to tough anti-terrorism policies might indeed be regarded as typical cases of the phenomenon of reciprocity, whose rational-choice foundations are increasingly understood (e.g. Matthew Rabin 1993, Ernst Fehr and Simon Gächter 1998, Gary Bolton and Axel Ockenfels 2000, Rajiv Sethi and E. Somanathan 2001 and 2003, Martin Dufwenberg and Georg Kirchsteiger 2004 and Armin Falk and Urs Fischbacher 2006). The second interpretation of provocation, namely 
dynamic inconsistency, stresses the effect that anti-terrorism policies can have on someone's personality and psychological state. The idea is here that someone who before a new policy comes into action (e.g. before the escalation of a war) still has no desire whatsoever to exercise violence - not even against tough policy makers, i.e. without reciprocal feelings ex ante - might nevertheless develop brutal preferences under the new policy environment. This is a typical example of developing a new self over time under new environments, resulting in dynamic inconsistency (e.g. Robert Strotz 1955-56, Peter Hammond 1976, Edward O'Donoghue and Matthew Rabin 1999, Roland Bénabou and Marek Pycia 2002). One might defend such an interpretation of provocation against one in terms of stable reciprocal preferences by invoking the radical and insane nature of the ex post attitude of creating terrorism: arguably, terrorists were not already born with the insane desire to perform terrorist attacks if future circumstances are such and such. This reasoning resembles that leading some economists to use dynamic inconsistency when modelling drug addiction: arguably, an addict was not already born with the insane desire that his future self, if addicted, consumes heroin. Psychological and empirical research will have to tell whether provocation by tough anti-terrorism policies reflects reciprocity or a dynamically inconsistent personality change; the answer might well be context- and person-specific. Importantly, our formal model is open to both explanations.

By standing in the tradition of the endogenous preference literature, this paper is little related to existing work on terrorism prevention in political economy. One branch of this field is empirical and investigates potential causes of terrorism (e.g. William Eubank and Leonard Weinberg 1994, Andrew Silke 1994, and Lloyd Dumas 2002). A more theoretical branch analyses concrete policy measures based on their efficiency in reducing terrorism and/or their costs, without incorporating provocation but accounting for several incentives and usually focussing on more specific problems than here (e.g. Claudio CioffiRevilla 1985 and 1998, Bruno Frey 2004, Walter Enders and Todd Sandler 2006). This empirical and theoretical literature provides several important insights not reviewed here; future research might combine them with the provocation effects studied here.

\section{Informal analysis}

Throughout the paper we consider a policy maker (e.g. a national government or international organisation) in charge of choosing and implementing an antiterrorism policy. The term 'anti-terrorism policy' is understood in a broad sense (made precise in Sections 2.2 and 2.3), possibly including social or educational institutions, military interventions, police presence, criminal legislation and jurisdiction, declarations and speeches by politicians, diplomatic relations or embargoes, and so on. 
The terrorism threat comes from the members of a (non-empty finite) set of individuals $N$, called the population. Crucially, $N$ contains not just individuals currently engaged in terrorist activities (arguably a frequent mistake) but also all potential ones. This speaks for a large definition of $N$ : it might include all humans on earth, or some group defined geographically, ethnically, religiously, or else. The term 'individual' always refers to members of $N$.

In response to the policy, each individual engages in some behaviour, which can be more or less violent; he $\mathrm{h}^{3}$ might exercise no violence at all, or perform small offences, or major terrorist attacks, and so on. I assume that an individual cares about two consequences of his behaviour: (i) a level of damage created, represented by a real number $x \geq 0$, and (ii) a level of punishment received, represented by another real number $y \geq 0$. The particular form punishment takes is irrelevant; it could be any personal disadvantage ('cost') incurred, such as having to hide from authorities (before or after damage creation), having to prepare the attack, or coming to prison afterwards. Usually, most individuals behave so as to obtain the no-damage-no-punishment outcome $(x, y)=(0,0)$. I use the term 'terrorist' resp. 'non-terrorist' in a technical sense to denote someone who causes positive damage $x>0$ resp. zero damage $x=0$ (without intending any further connotations that this sensitive terminology may have in normal language). Although I say throughout that someone 'chooses' his damage-punishment pair $(x, y)$, this pair is in fact the outcome not just of own behaviour but also of the policy: damage $x$ might depend on the level of protection of targets, and punishment $y$ on criminal legislation. (In practice, the outcome $(x, y)$ might further depend on chance, a case we could capture by re-interpreting $(x, y)$ as an expected-damage-expected-punishment pair. $\left.{ }^{4}\right)$

\subsection{Peaceful vs. brutal preferences}

Suppose a given policy is in action. As usual in economics, I assume that individual behaviour is guided by preference. Accordingly, let each individual $i$ in the population $N$ hold some preference order $^{5} \succeq \equiv_{i}$ on the set

$$
\mathbf{R}_{+}^{2}=[0, \infty) \times[0, \infty)=\{(x, y): x \geq 0, y \geq 0\} \text { (damage-punishment quadrant) }
$$

of damage-punishment pairs $(x, y)$; the associated relations of strict preference $\succ$ and indifference $\sim$ are defined as usual. ${ }^{6}$ As illustrated in Figure 1, some

\footnotetext{
${ }^{3}$ Throughout I use masculine pronouns, without intended gender restriction.

${ }^{4}$ To make the following analysis compatible with this re-interpretation, one would need two technical assumptions: (i) an individual's behaviour leads to a probability distribution on $\mathbf{R}_{+}^{2}$ that possesses a finite expectation in each coordinate; (ii) the individual ranks such probability distributions (behaviours) on the basis of their expectation pairs. Moreover, feasible sets (introduced below) should be re-interpreted as sets of feasible expectation pairs.

${ }^{5}$ Throughout, 'preference order' refers to a transitive and complete binary relation.

${ }^{6}$ For all $(x, y),\left(x^{\prime}, y^{\prime}\right) \in \mathbf{R}_{+}^{2}$, we have $(x, y) \succ\left(x^{\prime}, y^{\prime}\right) \Leftrightarrow\left[(x, y) \succeq\left(x^{\prime}, y^{\prime}\right)\right.$ and not $\left(x^{\prime}, y^{\prime}\right) \succeq$ $(x, y)]$, and $(x, y) \sim\left(x^{\prime}, y^{\prime}\right) \Leftrightarrow\left[(x, y) \succeq\left(x^{\prime}, y^{\prime}\right)\right.$ and $\left.\left(x^{\prime}, y^{\prime}\right) \succeq(x, y)\right]$.
} 
individuals might hold peaceful preferences, others brutal ones. Formally, I call a preference order $\succeq$ (on the damage-punishment quadrant $\mathbf{R}_{+}^{2}$ )

- peaceful if less damage is preferred to more ceteris paribus, i.e. if $(x, y) \succ$ $\left(x^{\prime}, y\right)$ whenever $x<x^{\prime}$, and brutal otherwise.
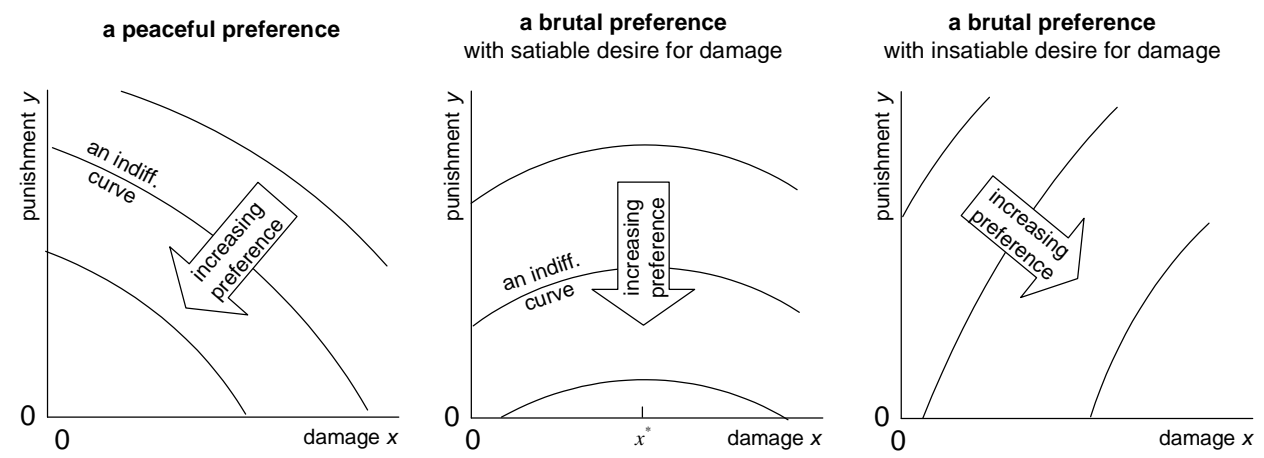

Figure 1: A peaceful and two brutal preferences (all three punishment-averse)

Someone's preference - whether peaceful or brutal - is usually punishmentaverse, where I call a preference order $\succeq$ (on the damage-punishment quadrant $\left.\mathbf{R}_{+}^{2}\right)$

- punishment-averse if less punishment is preferred to more ceteris paribus, i.e. if $(x, y) \succ\left(x, y^{\prime}\right)$ whenever $y<y^{\prime}$.

Given punishment-aversion (which I assume throughout the informal discussion), the difference between peaceful and brutal preferences shows in the slope of indifference curves: peaceful preferences have negatively sloped indifference curves (first plot in Figure 1), whereas brutal preferences have positively sloped indifference curves at least somewhere on the quadrant $\mathbf{R}_{+}^{2}$ (second and third plot in Figure 1). By anti-clockwise rotating the indifference curves, preference becomes more brutal, where I have in a natural way (partially) ordered the preference orders on $\mathbf{R}_{+}^{2}$ in terms of brutality:

- $\succeq$ is at least as brutal (or at most peaceful) as $\succeq^{\prime}$ if any preference for higher damage that holds under $\succeq^{\prime}$ also holds under $\succeq$, i.e. if $(x, y) \succeq^{\prime}$ $\left(x^{\prime}, y^{\prime}\right)$ and $x>x^{\prime}$ imply $(x, y) \succeq\left(x^{\prime}, y^{\prime}\right)$.

In this sense, the preference on the right of Figure 1 is more brutal than that in the middle, which is more brutal than that on the left. A radical form of brutal preferences $\succeq$ are ones with

- insatiable damage desire, i.e. $(x, y) \succ\left(x^{\prime}, y\right)$ whenever $x>x^{\prime}$,

in which case indifference curves are positively sloped on the entire quadrant $\mathbf{R}_{+}^{2}$ (third plot in Figure 1). (One might speculate whether some suicide bombers have insatiable damage desire.) The more moderate forms of brutal preferences are ones with a strictly positive but finite optimal damage level $x^{*}$ (which might depend on punishment $y$ ), where preference typically decreases as damage $x$ moves away from the optimum in either direction holding $y$ fixed (see second 
plot in Figure 1). For instance, someone might desire to destroy a building, but preferably without killing humans.

Each anti-terrorism policy results in some (non-empty) set $\mathbf{F} \subseteq \mathbf{R}_{+}^{2}$ of $f e a$ sible damage-punishment pairs from which individuals have to 'choose'. Figure 2 and later figures take the feasible set $\mathbf{F}$ to be 'thin' and linear and to render every damage level $x \geq 0$ feasible (nothing of which is essential ${ }^{7}$ ), with the plausible feature that punishment increases with damage and that no-damageno-punishment $(0,0)$ is feasible. Figure 2 shows how three types of individuals
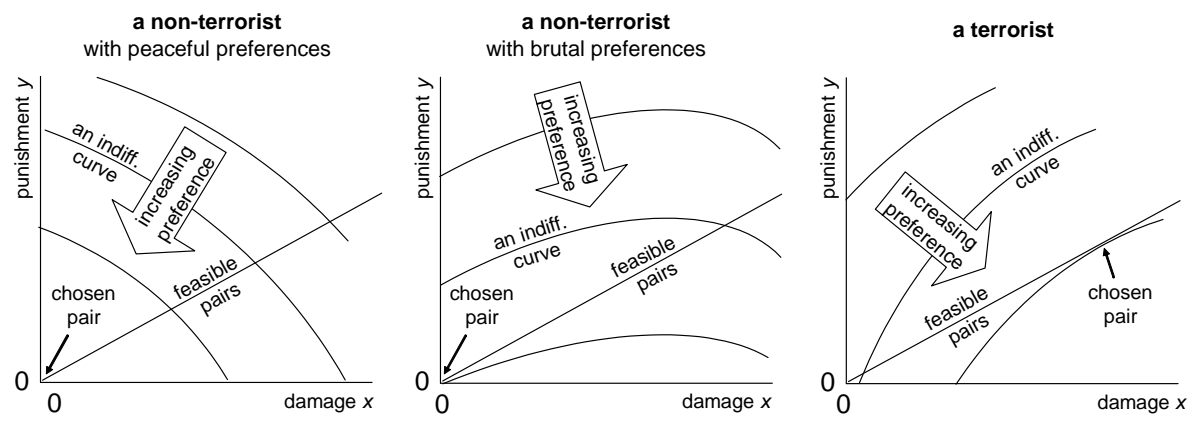

Figure 2: Behaviour of one peaceful and two brutal types under the policy

behave under the policy (all maximising preference): the peaceful type on the left creates no damage (is not a terrorist), the strongly brutal type on the right creates positive damage (is a terrorist), and the moderately brutal type in the middle creates no damage (but would have been a terrorist under only slightly more brutal preferences).

\subsection{Cause-related and symptom-related policy measures}

A policy measure can qualify as part of the anti-terrorism policy if, through whatever means, it affects the damage level created by individuals. Someone's damage level $x$ is determined by two factors: (i) his preference order $\succeq$ on the damage-punishment quadrant $\mathbf{R}_{+}^{2}$ and (ii) the feasible set $\mathbf{F} \subseteq \mathbf{R}_{+}^{2}$ from which he chooses. This naturally leads me to distinguish between two sorts of anti-terrorism policy measures, to be labelled 'cause-related' or 'symptomrelated' (without the derogative connotation that the term 'symptom-related' sometimes has in natural language):

- A cause-related or appeasement measure aims at changing the preferences of population members, not the constraints $\mathbf{F} \subseteq \mathbf{R}_{+}^{2}$ under which they can create terrorism. The goal is that brutal preferences become peaceful (as on the right in Figure 3) or at least less brutal (as on the left in Figure 3). Examples of such preference-appeasement attempts might be

\footnotetext{
${ }^{7}$ Feasible sets are discussed in full generality in Section 3.1.
} 
to improve education or the standard of living, reduce polarisation, allow people to sympathise with those persons, cultures or institutions against which terrorism might be directed, or render terrorism less desirable by reducing media attention as Bruno Frey (2004) advocates.
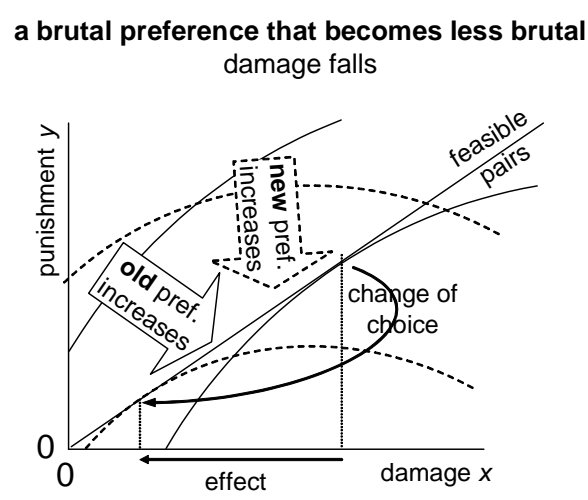

a brutal preference that becomes peaceful the person becomes a non-terrorist

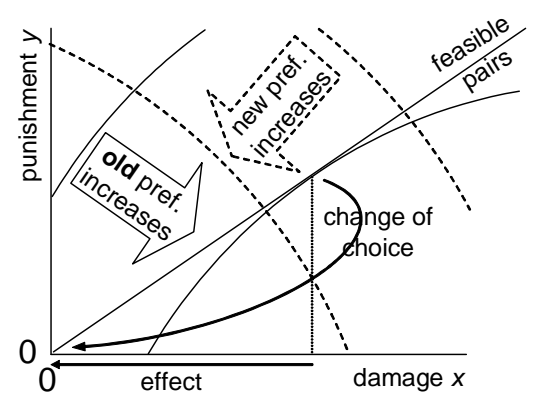

Figure 3: A succesful cause-related policy measure (new preference dashed)

- A symptom-related or deterrence measure aims to reduce terrorism by changing the constraints $\mathbf{F} \subseteq \mathbf{R}_{+}^{2}$ under which terrorists operate (possibly with brutalising side effects on people's preferences, as analysed in the next subsection). Roughly, such measures render the feasible set $\mathbf{F}$ 'steeper', and perhaps render some damage levels $x$ infeasible. ${ }^{8}$ Such measures can be defensive or aggressive. Defensive measures change the difficulty of creating damages $x$, for instance by erecting weapons embargoes, protecting buildings, supervising public places, enforcing transparency in bank transfers, or decentralising society to make it less vulnerable as Bruno Frey (2004) proposes. Aggressive measures change the kind or extent of punishment $y$, for instance by severe legislation, a worldwide search for terrorists, or a war. While there may be overlaps, the difference between defensive and aggressive deterrence can be formalised (see Section 4).

\subsection{Symptom-related measures and the problem of provo- cation (side) effects}

The rest of the paper (except Appendix A) analyses symptom-related policy measures as just defined. As Figure 4 illustrates, additional toughness does not lead to more terrorism if people's preferences are guaranteed to be nonprovocable, i.e. policy-invariant. However, as Figure 5 illustrates, symptomrelated policies often affect not just the set $\mathbf{F} \subseteq \mathbf{R}_{+}^{2}$ of feasible damage-punishment pairs but (as side effects) also some individuals' preferences. The same individual who in the status quo still holds peaceful preferences $\succeq$ on $\mathbf{R}_{+}^{2}$ (hence is not

\footnotetext{
${ }^{8}$ Damage level $x \geq 0$ is feasible if $(x, y) \in \mathbf{F}$ for some punishment $y \geq 0$. Some policies (e.g. weapons embargoes) render high damage levels infeasible; see Section 3.1.
} 

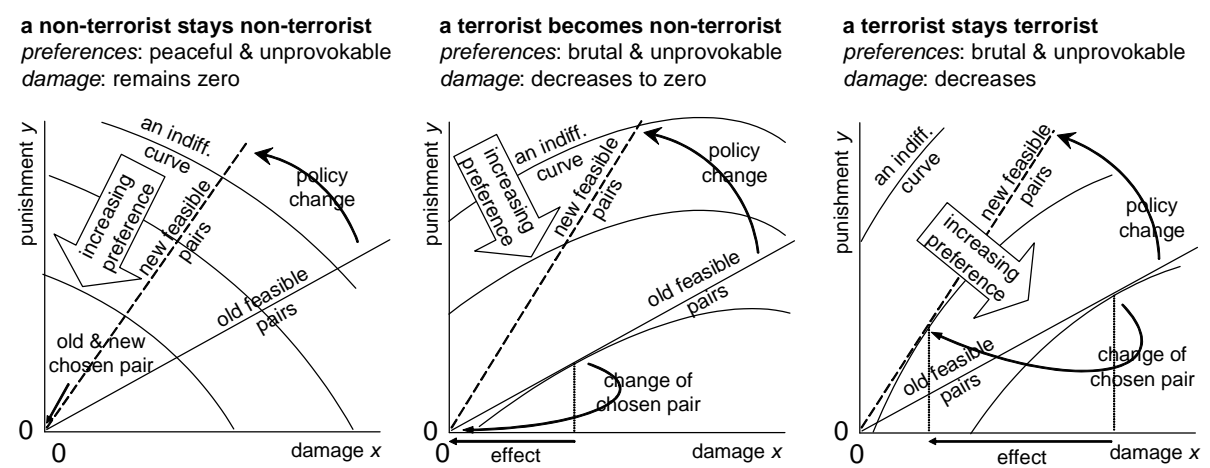

Figure 4: Effect of a toughness raise on three types of non-provocable individuals
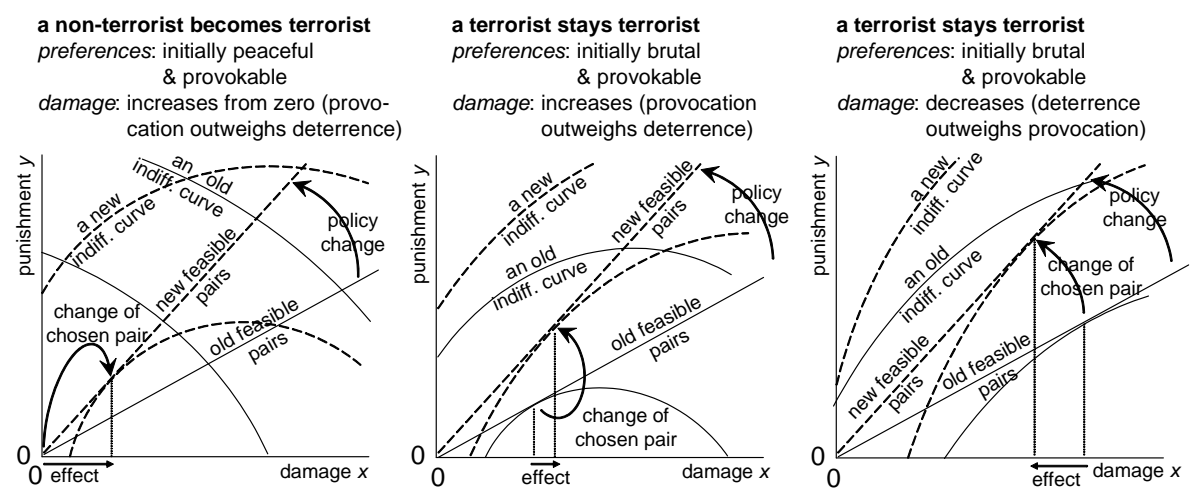

Figure 5: Effect of a toughness raise on three types of provocable individuals

a terrorist) might develop brutal preferences $\succeq^{\prime}$ on $\mathbf{R}_{+}^{2}$ under a new tougher policy. It is psychologically plausible and empirically observable that some persons' preference for or against exercising violence is not stable but reacts to the environment: some environments appease, others brutalise tastes and desires. The anti-terrorism policy may form part of the environment that shapes someone's personality and preferences. A policy may let someone develop hate feelings and a preference for creating damage, either in order to hurt the policy-makers themselves (if the hate feelings focus on them) or without a specific target (if the hate feelings are more diffuse). Such provocation is given two interpretations in Section 2.4, namely in terms of either dynamic inconsistency or extended preferences over damage-punishment-policy triples (the latter interpretation offering two perfectly rational explanations of provocation: reciprocity and taste acquisition).

This said, an individual's preference order on the damage-punishment quadrant $\mathbf{R}_{+}^{2}$ should be indexed by the policy $t$, say $\succeq_{t}$ with $t$ ranging over a set $T$ of relevant (symptom-related) policies among which the status quo policy $\bar{t}$. Here, $\succeq_{t}$ represents the individual's (more or less peaceful) dispositions under (the 
impression of) policy $t$. I call an individual, or the family of his policy-indexed preference orders $\left(\succeq_{t}\right)_{t \in T}$ on $\mathbf{R}_{+}^{2}$,

- unprovocable if preference $\succeq_{t}$ is the same for each policy $t \in T$;

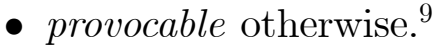

This definition of provocability leaves open the direction of the preference reaction. In principle, provocation could even take the inverse form that tough policies appease preferences (such as when someone becomes unable to touch a knife after the traumatic experience of a war); this psychological reaction, later referred to as 'inverse provocation', is possible but seems less frequent.

If one were to decompose someone's preferences into a damage-related utility and a punishment-related utility, say, through an additively separable utility model $u_{t}(x, y)=v_{t}(x)+w_{t}(y)$ as in Appendix A, then one is led to ask: does provocability come rather from damage utility $v_{t}(x)$ being policy-sensitive, or rather from punishment utility $w_{t}(y)$ being policy-sensitive? Certainly, utility derived from terrorism $v_{t}(x)$ seems more likely to be policy-sensitive: while the pain from a fixed punishment level $y$ seems policy-invariant, the pleasure of creating a fixed damage $x$ highly depends on how much the person dislikes the policy makers (cultures, etc.) he hurts, which may be policy-sensitive.

How does a policy toughening affect terrorism? As illustrated in Figure 4, the behaviour of unprovocable individuals is affected in a desirable way: nonterrorists stay non-terrorists, and terrorists reduce damage, possibly becoming non-terrorists. Such deterrence without provocation is extensively studied in the crime and terrorism literature, using different models. For provocable types, the picture changes and becomes less uniform. Damage increases for types where provocation outweighs deterrence (first two plots of Figure 5), but decreases for types where deterrence outweighs provocation (third plot in Figure 5). In Figure 6 , I decompose the total effect of the toughness rise on a terrorist's damage into

- a (usually negative) deterrence effect, representing the damage change if, hypothetically, preferences were to remain constant, and

- a (usually positive) provocation effect, caused by preference change.

There is no general rule as to which of these two competing effects is stronger; for the type on the left (right) in Figure 6, provocation (deterrence) is stronger. The same decomposition also works for non-terrorists: the deterrence effect is then zero (less than 'no damage' doesn't exist) but the provocation effect might be positive, turning the person into a terrorist.

I shall be formal in Section 3 about the decomposition into deterrence and provocation. But qualitatively, what can we learn already now? If the population is approximately homogeneous, the policy maker can reduce terrorism by adjusting the policy to the predominant type; for instance, high toughness is

\footnotetext{
${ }^{9}$ This terminology makes sense since $T$ consists of symptom-related policies. But if policies may differ also (or only) in cause-related measures (e.g. in the education system), the more general term '(un)changeable' is better than '(un)provocable'. Changing (appeasing) preferences is the whole point of cause-related anti-terrorism policies.
} 

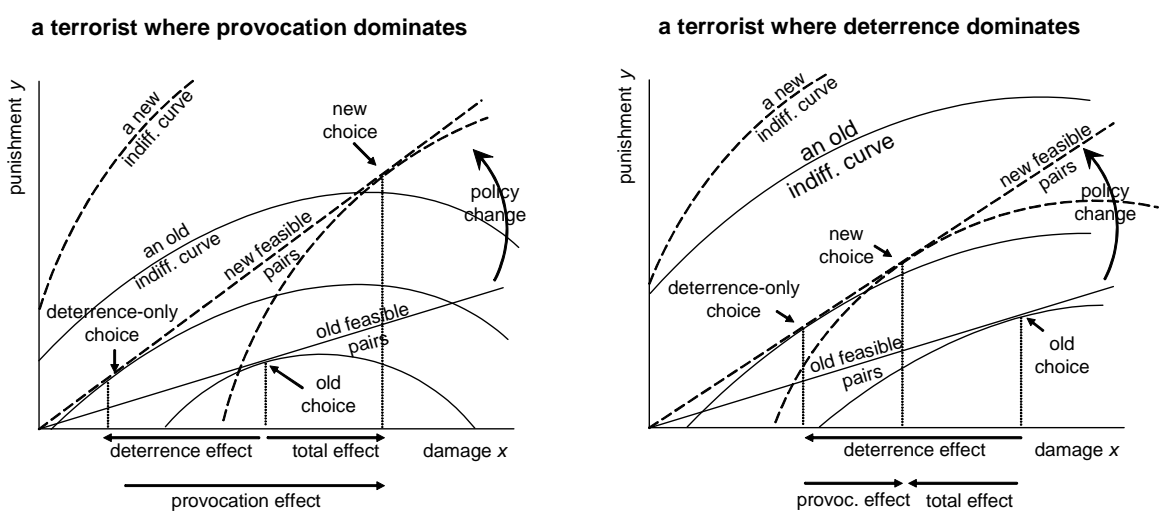

Figure 6: Deterrence effect and porvocation effect

efficient against a population dominated by types that are unprovocable (see Figure 4) or little provocable (see right plots in Figures 5 and 6). Often though, the population is significantly heterogeneous and contains many types (preferences), some more provocable than others, some peaceful and others brutal in the status quo. Then the policy maker faces the difficult task of finding the right compromise given the type distribution. As a rule of thumb, optimal toughness is decreasing as a function of the level of provocability and increasing as a function of the level of (status quo) brutality in the type distribution. More precisely, a policy shift from the status quo $\bar{t}$ to some new policy $t \in T$ minimises sum-total terrorism, as given by the sum $\mathbf{x}=\sum_{i \in N} x_{i}$ of damage levels $x_{i}$ across individuals $i \in N$, if and only if it minimises the policy's aggregate effect on damage (i.e. the change of $\mathbf{x}$ from the status quo). This effect can be decomposed into the sum $\mathbf{D E}(t)+\mathbf{P E}(t)$ of the aggregate deterrence effect $\mathbf{D E}(t)$ and the aggregate provocation effect $\mathbf{P E}(t) .{ }^{10} \mathbf{D E}(t)$ and $\mathbf{P E}(t)$ measure how much policy $t$ affects terrorism through deterrence and provocation, respectively. In practice, the policy maker faces two distinct challenges:

Finding the right level of toughness. Suppose the policy maker decides on a single policy parameter (e.g. the size of a military intervention), so that we can identify policies with toughness levels $t$ chosen from a one-dimensional policy space $T \subseteq \mathbf{R}$. As Figure 7 illustrates, in trying to minimise the function $\mathbf{D E}(t)+\mathbf{P E}(t)$, the policy maker faces a standard one-dimensional trade-off because $\mathbf{D E}(t)$ is decreasing but $\mathbf{P E}(t)$ increasing in toughness $t$ (for details, see Section 3). A minimum of $\mathbf{D E}(t)+\mathbf{P E}(t)$ defines an optimum on the trade-off between deterrence and provocation. Overshooting toughness increases terrorism by provoking too much, and undershooting toughness increases terrorism

\footnotetext{
${ }^{10}$ That is, $\mathbf{D E}(t)=\sum_{i \in N} D E_{i}(t)$ where $D E_{i}(t)$ is individual $i$ 's deterrence effect, defined as his change of damage holding his preferences fixed, i.e. neglecting provocation. Similarly, $\mathbf{P E}(t)$ is the sum of the individual provocation effects $P E_{i}(t), i \in N$.
} 


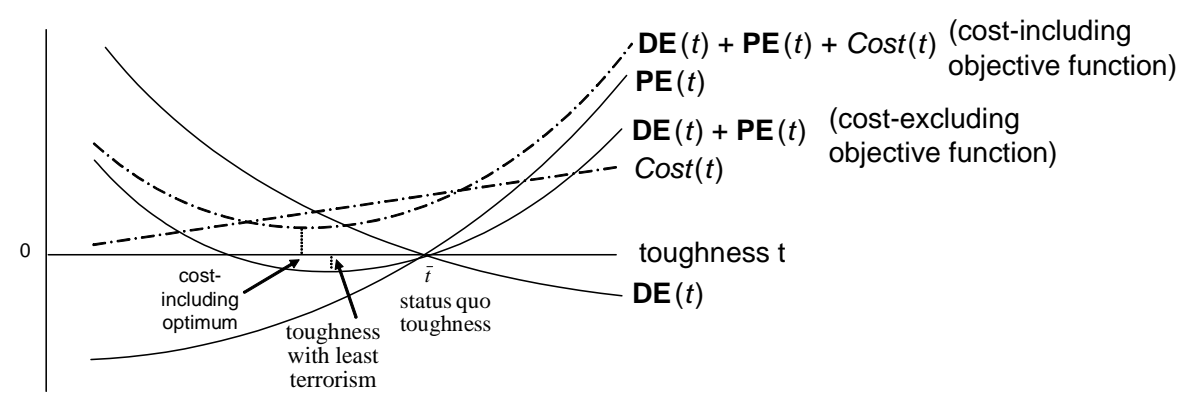

Figure 7: An example in which reducing toughness reduces terrorism

by deterring too little. As also illustrated in Figure 7, a refined objective function might be $\mathbf{D E}(t)+\mathbf{P E}(t)+\operatorname{Cos} t(t)$, where $\operatorname{Cos} t(t)$ represents the (suitably scaled) cost of toughness $t$ (such as financial costs, loss of human lives, and loss of life quality through state supervision). As Cost $(t)$ typically increases in toughness $t$, the optimum is typically reached at lower toughness than under the cost-neglecting objective function $\mathbf{D E}(t)+\mathbf{P E}(t)$. In short, toughness $t$ should be the lower, the steeper the $\mathbf{P E}(t)$ curve is (more provocability), the flatter the $\mathbf{D E}(t)$ curve is (less deterrability), and the steeper the cost curve $\operatorname{Cost}(t)$ is (more costly toughness).

Finding the right kind of toughness. Suppose now that a policy $t$ is given by many policy parameters: criminal legislation, weapons embargoes, police presence, military interventions, and so on. This may be represented by a multidimensional policy space $T .{ }^{11}$ Interestingly, the same level of deterrence - i.e. the same set $\mathbf{F}$ of feasible damage-punishment pairs, hence the same deterrence effect $\mathbf{D E}(t)$ - is often achievable by several policies $t \in T$ that differ in their dimension-specific toughness levels and thereby provoke in different ways and to different overall extents $\mathbf{P E}(t)$ : some of these policies may lead to peaceful preferences on $\mathbf{R}_{+}^{2}$ for most individuals, others to many brutal preferences. On which dimensions should the policy be tough, on which mild? Our model recommends a policy that achieve its overall level of deterrence in the least provoking way, which is implemented by allocating toughness to dimensions where deterrence comes with little provocation. The reason is that different policies $t$ with same aggregate deterrence effect $\mathbf{D E}(t)$ can be compared based just on their aggregate provocation effect $\mathbf{P E}(t)$ (possibly plus policy costs $\operatorname{Cos}(t)$ ). For instance, if introducing a weapons embargo leaves most individuals' preferences either totally unchanged (as in Figure 3) or brutalises them just slightly in the sense that deterrence overweighs provocation (as in the right plots of Figures 5 and 6 ), then this toughness raise is desirable, and preferable to other

\footnotetext{
${ }^{11}$ That is, $T \subseteq \mathbf{R}^{k}$, where a policy is seen as a vector $t=\left(t_{1}, \ldots, t_{k}\right)$, and $t_{1}$ is the level of toughness of $1^{\text {st }}$ kind, $t_{2}$ that of $2^{\text {nd }}$ kind, and so on.
} 
ones that deter equally but provoke more. ${ }^{12}$ Whether overall deterrence should be high (i.e. F should be 'steep') is context-dependent.

\subsection{Provocable preferences: a case of rationality or of dynamic inconsistency?}

What can make someone's preference over damage-punishment pairs react to the policy (e.g. to a war)? I deliberately leave the paper's analysis compatible with two classical economic interpretations of preference change: dynamic inconsistency, and what I call rational taste change in deference to Gary Becker's terminology.

Rational taste change: reciprocity and acquired tastes. Under this interpretation, an individual's policy-dependent preference orders $\succeq_{t}, t \in T$, (on the damage-punishment quadrant $\mathbf{R}_{+}^{2}$ ) are derived from a single stable extended preference order $\check{\succeq}$ over the set $\mathbf{R}_{+}^{2} \times T$ of damage-punishment-policy triples $(x, y, t)$, in which $t$ plays the role of a taste parameter: for each $t \in T,(x, y) \succeq_{t}$ $\left(x^{\prime}, y^{\prime}\right)$ then simply means that $(x, y, t) \check{\succeq}\left(x^{\prime}, y^{\prime}, t\right)$, i.e. that the individual prefers having $(x, y)$ with policy $t$ to having $\left(x^{\prime}, y^{\prime}\right)$ with policy $t$ (just as someone might prefer white to red wine with desert, though perhaps not with cheese). Such extended preferences can rationalise provocation, in at least two ways. First, there may be a desire to reciprocate, i.e. to be violent to tough policy makers (foreigners, etc.) and peaceful to mild ones. Second, the ability to enjoy terrorism may be acquired, namely through experiencing the policy, e.g. a war (just as a consumer à la Becker acquires the ability to enjoy a good by building up a stock of social and personal capital); note that taste acquisition does not imply dynamic inconsistency (just as the Becker consumer is not dynamically inconsistent: he anticipates his future abilities). The indifference curves of $\succeq_{t}$ (plotted in our figures) are derived from the higher-dimensional indifference sets of $\tilde{\succeq}$ (in the space $\mathbf{R}_{+}^{2} \times T$ ) by fixing the 'third coordinate' $t$, i.e. by intersecting with the subspace $\mathbf{R}_{+}^{2} \times\{t\}$.

Dynamic inconsistency. A dynamically inconsistent agent disapproves of his own future preference: he undergoes a personality change under the impression of the changing environment (policy), and his preference change is not representable by stable extended preferences (or stable intertemporal preferences over complete event streams). To illustrate, suppose the policy changes from the status quo 'mild' ( $\bar{t}$ ) to 'tough' $(t)$. How would someone whose preference changes from 'peaceful' $\left(\succeq_{\bar{t}}\right)$ to 'brutal' $\left(\succeq_{t}\right)$ describe himself before the change?

- In the earlier case of rational taste change, he might say: "I want and will always want to harm tough foreigners and to be kind to mild ones, and

\footnotetext{
${ }^{12}$ The embargo does indeed deter: the feasible set $\mathbf{F} \subseteq \mathbf{R}_{+}^{2}$ gets 'steeper' because damage creation gets harder (if feasible at all) and more criminal (so more highly punished).
} 
so I am currently not a terrorist but intend to become one whenever the foreigners becomes tough."

- In the case of dynamic inconsistency, he might say: "I am currently a pacifist who does not want to harm any mild or tough foreigners, and I wish I could prevent that, once foreigners become tough, my personality changes and I develop a desire to harm them."

More formally, the person's present preference about his future damagepunishment under future policy $t$ are given by:

- present taste $\succeq_{\bar{t}}$ in the case of dynamic inconsistency;

- future taste $\succeq_{t}$ in the case of rational taste change;

- some combination of $\succeq_{\bar{t}}$ and $\succeq_{t}$ in mixed cases.

Empirically, the difference between these kinds of preference change is revealed in commitment behaviour. ${ }^{13}$ In economics, dynamic inconsistency is often associated with individuals whose mental state is subjected to chocks or influences, either of an external kind (brutal friends, war) or an internal kind (Alzheimer, puberty). In this sense, provocation seems a natural candidate for dynamic inconsistency.

Which explanation of provocation, then, is appropriate? Answers are likely to be both context-dependent and controversial; the reader might choose his or her preferred explanation. It might even be that (within the same application) some individuals undergo a rational taste change and others a dynamic inconsistency. It is thus important that our model of provocation is not committed to one interpretation only.

The origin of provocation matters in at least two ways. First, it may determine the manner in which provocability should be empirically measured or tested. Second, it becomes behaviourally relevant in extensions of our model. Why so? The present model can leave the question open essentially because each individual gets to choose only once a damage-punishment pair, namely after the new policy is implemented, and so only his then-preference matters for behaviour, regardless of how it came about. However, in an extended model of repeated interaction between policy maker and population, the origin of provocation affects individual strategies, hence optimal policies to prevent terrorism.

\subsection{The fallacy of neglecting provocation}

Provocation effects of policies are easy to overlook in practice, for systematic reasons given in a moment. Probably they are being overlooked or underestimated in contemporary anti-terrorism politics, but this is an empirical claim

\footnotetext{
${ }^{13}$ Dynamically inconsistent (forward-looking) agents may choose to commit themselves, even if commitment comes with a cost. E.g. Robert Strotz (1955-56) and Peter Hammond (1976).
} 
that this theoretical paper cannot defend. Rather, let me briefly discuss (i) consequences of and (ii) explanations for provocation-neglect.

Consequence of provocation-neglect: toughness exaggeration. (The rigorous treatment comes in Section 3.4.) A provocation-neglecting policy maker minimises the wrong objective function since he wrongly predicts people's response to the policy. He assumes people keep their old (status quo) preferences under the new policy: peacefully minded persons stay peacefully minded, brutally minded persons stay equally brutally minded. This leads the provocationneglecting policy maker to minimise $\mathbf{D E}(t)+\operatorname{Cos} t(t)$ rather than $\mathbf{D E}(t)+$ $\mathbf{P E}(t)+\operatorname{Cos}(t)$, where, as in Section 2.3, $\mathbf{D E}(t), \mathbf{P E}(t)$ and Cost $(t)$ denote the aggregate deterrence effect, the aggregate provocation effect, and the cost of policy $t \in T$, respectively. Since $\mathbf{P E}(t)$ is an increasing function of toughness

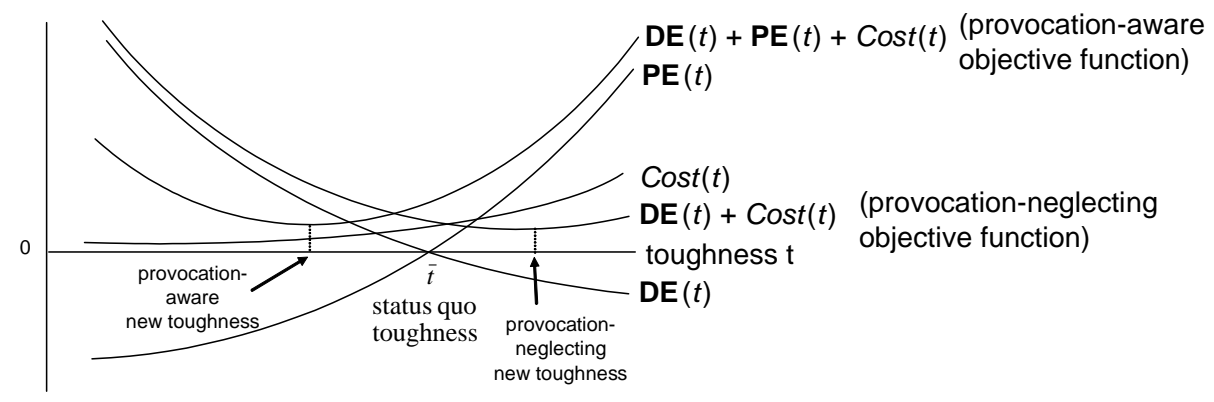

Figure 8: An example in which provocation-neglect leads to a toughness raise, but provocation-awareness to a toughness reduction

(see Theorem 1 below), the provocation-neglecting policy maker is tougher than would be optimal, as Figure 8 illustrates in the case of a one-dimensional policy choice problem. Such toughness exaggeration due to provocation-neglect may be called the 'fallacy of neglecting provocation'.

Explaining provocation-neglect. Is provocation-neglect an elementary mistake that cannot be expected to occur among policy makers? On the contrary, provocation-neglect may be a tempting error, almost like a trap wide open in front the policy maker who can avoid it only by particular serenity. The reason is that people's provocability may be little visible before the new policy (e.g. before a war): it is not (yet) revealed in behaviour, perhaps not even in speech, especially if provocation comes from dynamic inconsistency (see Section 2.4). In a relatively mild status quo environment, individuals who would develop brutal preferences if the policy were toughened may display perfectly sane and harmless behaviour, and even declare the peacefulness of their intensions. It certainly takes special serenity and psychological and cultural sensitivity to foresee if and how a policy would provoke those subgroups who so far behave peacefully and 
whose (dynamically inconsistent) members are perhaps even themselves unaware of being provocable.

Do there exist indirect ways to nevertheless 'observe' or 'test' beforehand if and how people would be provoked by new policies? In now briefly address this question.

First, consider speech-revelation: can one trust someone's speech about what he would desire or do under such and such new policy? Speech-revealed preferences, which many economists legitimately treat with caution, may be particularly unreliable here, for two reasons:

- A person might not sincerely reveal the brutality of his future preferences or actions, by fear of the consequences of making his criminal side known.

- We are dealing here with revealing not the present preference order (over damage-punishment pairs), but future preferences held under potential new policies. Revealing these is in principle possible, and might be realistic under the reciprocity interpretation because then the future desires already exist presently in the form of conditional preferences. But the plausibility of revealing future preferences decreases if these preferences arise by dynamic inconsistency (or by rationally acquired tastes): the person might then not be aware that a new environment would turn him into a terrorist, also given that this drastic event would presumably be unprecedented in his life.

Second, while speech-revelation is thus limited as a tool to 'measure' provocability, certain past observations may serve as proxis to estimate provocability. Similar policies might in the past have been used in similar contexts and on similar populations (though many culture- and context-specific factors would have to be controlled for). Also, the population might in the past have displayed certain (more or less violent) behavioural patterns in response to environments (such as more or less rough social environments) that, though not identical to the policy-induced environments, resemble them. In the best case scenario, past data allow one to statistically estimate, for each policy $t \in T$, the population's resulting distribution of preferences $\succeq_{t}$.

\section{Formal analysis}

The above informal analysis draws on some claims, in particular about the signs of two competing effects of toughness on terrorism: the provocation effect is typically non-negative, the deterrence effect typically non-positive. But what means 'typically'? I take this question up now by giving sufficient conditions for these claims to hold. A social welfare analysis of toughness will also confirm the earlier claim that provocation-neglect leads to toughness exaggeration.

To draw a comparison first, Slutsky's fundamental equation of demand theory decomposes the effect of a price increase on demand into two conceptually 
distinct effects, the income effect and a substitution effect. We pursue a similar goal in decomposing the effect of toughness on terrorism (damage) into two conceptually distinct effects, deterrence and provocation. In spite of obvious differences, our approach shares some key aspects with Slutsky's:

(i) The primary level of description is the individual: the effect of a price/toughness raise on overall demand/terrorism is obtained by aggregating the effect on individual demand/terrorism. Accordingly, much of this section focusses on a single individual, with the understanding that one could later aggregate.

(ii) The effect of a price/toughness change can be analysed either by comparing the status quo price/toughness with a fixed new price/toughness, as done in textbook discussions of Slutsky's decomposition and in Section 2, or by considering a marginal price/toughness change, i.e. by differentiating demand/terrorism with respect to price/toughness, as done in Slutsky's equation and in this section.

(iii) Each subeffect has a typical sign, yet there are exceptions, such as income effects getting positive for inferior goods and provocation effects getting negative for inversely provocable individuals.

(iv) The two subeffects are constructed by means of a thought experiment involving hypothetical behaviour: the substitution (resp. deterrence) effect represents how a price (resp. toughness) change would affect demand (resp. terrorism) if, hypothetically, the individual's achieved utility level (resp. his preference order $\succeq$ ) did not change.

\subsection{Framework, terminology, notation}

Throughout we consider the following sequence of events. First the policy maker chooses a policy $t$ from a given set $T$ of possible policies, among which the status quo policy $\bar{t}$. Each policy $t \in T$ leads to a set $\mathbf{F}_{t} \subseteq \mathbf{R}_{+}^{2}$ of feasible damage-punishment pairs $(x, y)$. Under the environment of policy $t \in T$, each individual $i$ in the (finite non-empty) population $N$ holds some (complete and transitive) preference order $\succeq_{t, i}$ on the damage-punishment quadrant $\mathbf{R}_{+}^{2}$ that guides his behaviour, i.e. his choice of a damage-punishment pair $(x, y)$ from the feasible set $\mathbf{F}_{t}$. I call the policy maker provocation-aware (resp. -neglecting) if he believes that any policy $t \in T$ gives any individual $i$ the true preferences $\succeq_{i, t}$ (resp. the status quo preference $\succeq_{i, \bar{t}}$ ).

In spite of what the figures in Section 2 might suggest, feasible sets $\mathbf{F}_{t}$ need neither be linear, nor be 'thin', nor render all damage levels $x \in \mathbf{R}_{+}$feasible. In general, each feasible set has two by-products: the feasible damage set and the punishment function, defined and denoted as follows. Policy t's feasible damage set $\mathbf{X}_{t} \subseteq \mathbf{R}_{+}$is defined as $\mathbf{X}_{t}:=\left\{x:(x, y) \in \mathbf{F}_{t}\right.$ for some $\left.y \in \mathbf{R}_{+}\right\}$(the projection of $\mathbf{F}_{t}$ on the $x$-coordinate), representing what damage is physically possible under policy $t$. If $\mathbf{X}_{t}=\mathbf{R}_{+}$, any damage can be created. If $\mathbf{X}_{t} \subsetneq \mathbf{R}_{+}$, the policy physically limits the kind or extent of damage people can create, for 
instance by weapons embargoes or police presence or airport controls, which make major terrorist attacks simply impossible to create, thus leaving only the more 'modest' targets for terrorists. Typically, the feasible damage set $\mathbf{X}_{t}$ contains at least $x=0$ ('no damage' is feasible) and forms an interval, which could be unbounded $\left(\mathbf{X}_{t}=\mathbf{R}_{+}\right)$or bounded $\left(\mathbf{X}_{t}=\left[0, x_{t}^{*}\right]\right.$ or $\mathbf{X}_{t}=\left[0, x_{t}^{*}\right)$ where $x_{t}^{*}$ is a feasibility bound established by policy $t$ ). Policy $t$ 's punishment function $f_{t}: \mathbf{X}_{t} \rightarrow \mathbf{R}_{+}$maps every feasible damage level $x \in \mathbf{X}_{t}$ to the minimally received punishment, i.e. $f_{t}(x):=\inf \left\{y:(x, y) \in \mathbf{F}_{t}\right\}$. The graph of the function $f_{t}$ represents the southern border of the feasible set $\mathbf{F}_{t}$. An important example are feasible sets of the ('thin') form

$$
\mathbf{F}_{t}=\left\{\left(x, f_{t}(x)\right): x \in \mathbf{X}_{t}\right\}, t \in T
$$

consisting of pairs of a feasible damage $x \in \mathbf{X}_{t}$ and a unique punishment $f_{t}(x)$; here each feasible set $\mathbf{F}_{t}$ is 'thin' in that it coincides with the graph of $f_{t}$.

Typically, a policy $t$ 's punishment function $f_{t}$ is increasing: higher punish-

ment for higher damage. Intuitively, the tougher the policy $t$, the higher the punishments $f_{t}(x), x \in \mathbf{X}_{t}$, and also the fewer the feasible damage levels, i.e. the smaller $\mathbf{X}_{t}$. However, it is perfectly possible for two policies in $T$ that one gives higher punishment yet renders more damage levels feasible, or that one gives more punishment for some damage levels but less for others; then these two policies cannot easily be ranked in terms of their toughness or deterrence.

In practice, individuals often have many ways to produce a given damage $x \geq 0$ (e.g. many ways to kill someone), and punishment might depend on the chosen way. Feasible sets do then not take the 'thin' form (1) but the general form

$$
\mathbf{F}_{t}=\left\{(x, y) \in \mathbf{R}_{+}^{2}: y \in Y_{t}(x)\right\}, t \in T,
$$

where, for each policy $t \in T$ and each damage level $x \geq 0, Y_{t}(x)$ is a set $Y_{t}(x) \subseteq \mathbf{R}_{+}$of punishment levels that can occur in combination with damage level $x$. In fact, feasible sets $\mathbf{F}_{t}, t \in T$, can always be written in the latter form; in the 'thin' case (1), each set $Y_{t}(x)$ is singleton (if $x$ is feasible) or empty (if $x$ is infeasible).

\subsection{Marginal provocation effect and deterrence effect}

In the rest of Section 3 (but not in Appendix A), the policy maker chooses a single policy parameter representing the toughness level. More precisely:

Unidimensional Policy Space UP. The set of policies $T$ is an interval $T \subseteq \mathbf{R}$ (of toughness levels), and the status quo $\bar{t} \in T$ is non-extremal, i.e. not on the boundary of the interval $T$.

We consider an individual whose preferences $\succeq_{t}, t \in T$, are regular as defined by three conditions: 
R1 (punishment-aversion) For every policy $t \in T, \succeq_{t}$ is punishment-averse, i.e. $(x, y) \succ_{t}\left(x, y^{\prime}\right)$ whenever $y<y^{\prime}$.

R2 (continuity) For every policy $t \in T, \succeq_{t}$ is continuous, hence is (by Debreu's Theorem) representable by a continuous utility function $u_{t}: \mathbf{R}_{+}^{2} \rightarrow \mathbf{R}$.

R3 (unique optimum) For all policies $t_{1}, t_{2} \in T$, there exists a unique damagepunishment pair, denoted $\left(\mathrm{x}\left(t_{1}, t_{2}\right), \mathrm{y}\left(t_{1}, t_{2}\right)\right)$, that maximises $\succeq_{t_{1}}$ within $\mathbf{F}_{t_{2}}$ (i.e. that is an optimal response to policy $t_{2}$ under the preferences of policy $\left.t_{1}\right)$, and moreover the damage level $\mathrm{x}\left(t_{1}, t_{2}\right)$ is a differentiable function of $\left(t_{1}, t_{2}\right) \in T \times T{ }^{14}$

The optimisation problem in R3 is hypothetical in that under policy $t_{2}$ the individual really maximises $\succeq_{t_{2}}$, not $\succeq_{t_{1}}$; but if we set $t_{1}=t_{2}=t$, we obtain precisely the individual's real optimisation problem under policy $t$. Hence, R3 in particular implies that

- to each policy $t \in T$ the individual has a unique optimal response, to be denoted $(\mathrm{x}(t), \mathrm{y}(y))(=(\mathrm{x}(t, t), \mathrm{y}(t, t)))$.

So we can define the marginal effect of raising toughness from the status quo $\bar{t}$ :

- The (marginal) effect (of toughness) is defined as $E:=\mathrm{x}^{\prime}(\bar{t})$, the derivative (at the status quo) of damage with respect to toughness. ${ }^{15}$

How can we meaningfully decompose $E$ into two subeffects? As illustrated

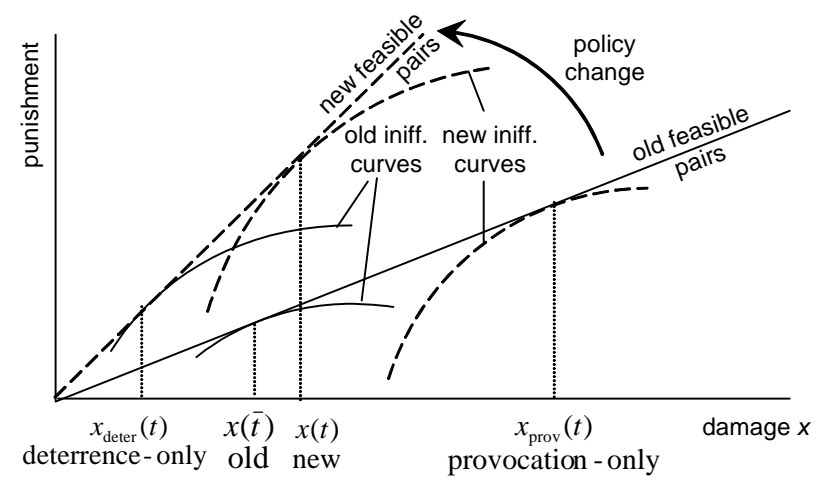

Figure 9: The old, the new, and the two hypothetical damage levels

in Figure 9, the key is to first introduce two hypothetical behaviours, one that neglects provocation and one that neglects deterrence:

- The pure-deterrence or provocation-neglecting response to policy $t \in T$, denoted $\left(\mathrm{x}_{\text {deter }}(t), \mathrm{y}_{\text {deter }}(t)\right)$, is defined as $(\mathrm{x}(\bar{t}, t), \mathrm{y}(\bar{t}, t))$, the choice that

\footnotetext{
${ }^{14}$ Throughout, the derivative of a function at a point on the boundary of the function's domain is interpreted as usual, i.e. as a one-sided derivative.

${ }^{15} \mathrm{R} 3$ ensures that $\mathrm{x}(t)$ (and $\mathrm{x}_{\mathrm{deter}}(t)$ and $\mathrm{x}_{\mathrm{prov}}(t)$ defined below) are indeed differentiable functions. See the proof of Theorem 1 .
} 
maximises the old preference $\succeq_{\bar{t}}$ within the new feasible set $\mathbf{F}_{t}$. It captures deterrence without provocation, as it represents how the individual would react to the new policy if (hypothetically) his preferences were to remain unchanged. It represents how a provocation-neglecting policy maker predicts the individual's response to policy $t$.

- The pure-provocation or deterrence-neglecting response to policy $t \in T$, denoted $\left(\mathrm{x}_{\text {prov }}(t), \mathrm{y}_{\text {prov }}(t)\right)$, is defined as $(\mathrm{x}(t, \bar{t}), \mathrm{y}(t, \bar{t}))$, the choice that maximises the new preference $\succeq_{t}$ within the old feasible set $\mathbf{F}_{\bar{t}}$. It captures provocation without deterrence, by representing how the individual would react to the new policy $t$ if (hypothetically) he did not yet face the new constraints (such as new punishment levels). Under another interpretation, it represents the individual's reaction if, although his preferences are already affected (perhaps provoked) by the new environment, he is short-sighted or irrational in that he ignores the new punishment levels he faces.

The pure-deterrence damage $\mathrm{x}_{\text {deter }}(t)$ and pure-provocation damage $\mathrm{x}_{\text {prov }}(t)$ represent two partial views on the person's damage response to policy $t: \mathrm{x}_{\text {deter }}(t)$ is optimal under old preferences given new punishment levels, and $\mathrm{x}_{\text {prov }}(t)$ is optimal under new preferences supposing old punishment levels. I can now define deterrence and provocation effects.

- The (marginal) deterrence effect (of toughness) is defined as $D E:=\mathrm{x}_{\mathrm{deter}}^{\prime}(\bar{t})$, the derivative (taken at the status quo $t=\bar{t}$ ) of the pure-deterrence damage $\mathrm{x}_{\text {deter }}(t)$. It captures the marginal damage change as far as it is caused by changing constraints (punishments), ignoring any meanwhile preferences change.

- The (marginal) provocation effect (of toughness) is defined as PE := $\mathrm{x}_{\text {prov }}^{\prime}(\bar{t})$, the derivative (taken at the status quo $t=\bar{t}$ ) of the pureprovocation damage $\mathrm{x}_{\text {prov }}(t)$. It captures the marginal damage change as far it is caused by preference change, ignoring the changing constraints (punishments).

\subsection{Theorem}

I now show that the total effect of toughness is decomposable into $E=P E+D E$ with $P E \geq 0$ and $D E \leq 0$. While the additive decomposition $E=P E+D E$ is simple to prove (essentially, by applying the chain rule), the claim on the signs of the subeffects is non-trivial and does not hold universally, but under meaningful conditions. Specifically, each inequality, $P E \geq 0$ and $D E \leq 0$, is based on exactly one condition on preferences. The condition for $P E \geq 0$ excludes that raising toughness appeases the preference; more precisely:

Condition NIP (no inverse provocability) If the individual is currently indifferent between two damage-punishment pairs, then a toughness raise cannot 
make him prefer the pair with lower damage. That is, whenever $(x, y) \sim_{\bar{t}}\left(x^{\prime}, y^{\prime}\right)$ with $x<x^{\prime}$, then no policy $t \in T$ with $t>\bar{t}$ leads to $(x, y) \succ_{t}\left(x^{\prime}, y^{\prime}\right)$.

NIP is plausible - it is less demanding than requiring that $\succeq_{t}$ be at least as brutal as $\succeq_{\bar{t}}$ (see Section 2.1) whenever $t>\bar{t}$ - but not universal: surely, there also exist inversely provocable individuals, such as ones who after the traumatic experience of a war lose any desire to exercise violence themselves. For such individuals, the provocation effect $P E$ can become negative.

As the deterrence effect $D E$ is (unlike $P E$ ) defined by holding preferences fixed, the inequality $D E \leq 0$ has to be based on a condition quite different to NIP: not a condition about how preference changes as the policy changes, but one about internal consistency of status quo preference:

Condition TC (translation-consistency) Under the status quo preferences, a preference of one damage-punishment pair over another with lower punishment is not reversed by any symmetric punishment increase. That is, whenever $(x, y) \succ_{\bar{t}}\left(x^{\prime}, y^{\prime}\right)$ with $y>y^{\prime}$, then for no $\epsilon>0$ there is $(x, y+\epsilon) \prec_{\bar{t}}\left(x^{\prime}, y^{\prime}+\epsilon\right)$.

By TC, an extra amount of punishment cannot hurt less if the person already suffers more punishment; for instance, an extra hour of compulsory labour cannot hurt less if it comes on top of 10 hours than if it comes on top of 5 hours. $\mathrm{TC}$ is again plausible but not universal, and its failure can render the deterrence effect $D E$ positive.

Unlike the definition of $P E$, that of $D E$ is based on varying the feasible set $\mathbf{F}_{t}$, and so the sign of $D E$ cannot possibly be independent of how $\mathbf{F}_{t}$ reacts to the policy $t \in T$. This is why the inequality $D E \leq 0$ requires an extra condition on feasible sets, one that relates the shape of $\mathbf{F}_{t}$ to the toughness level $t \in T$. Specifically, I require that the tougher the policy $t \in T$ is, the 'steeper' the feasible set $\mathbf{F}_{t}$ becomes, i.e. the larger marginal punishment becomes:

Condition MP (marginal punishment increases with toughness) Each feasible set $\mathbf{F}_{t}, t \in T$, contains the no-damage-no-punishment pair $(0,0)$, it is (topologically) closed and connected, and its marginal punishment function $f_{t}^{\prime}: \mathbf{X}_{t} \rightarrow \mathbf{R}$ is defined ${ }^{16}$, non-negative, and (at least weakly) increasing in toughness $t^{17}$.

Essentially, MP requires the southern border of the feasible set $\mathbf{F}_{t}$ (i.e. the graph of $f_{t}$ ) to have a non-negative slope that increases if toughness $t$ increases. In the special case that each feasible set $\mathbf{F}_{t}$ is 'thin' (i.e. identical to its southern border: $\left.\mathbf{F}_{t}=\left\{\left(x, f_{t}(x)\right): x \in \mathbf{X}_{t}\right\}\right)$, MP simply requires feasible sets to everywhere have a non-negative slope that increases with toughness. MP holds for instance if $T=(0, \infty)$ and each $\mathbf{F}_{t}$ has southern border of

\footnotetext{
${ }^{16}$ That is, the punishment function $f_{t}: \mathbf{X}_{t} \rightarrow \mathbf{R}_{+}$is differentiable.

${ }^{17}$ That is, $t<t^{\prime}$ implies that $f_{t}^{\prime}(x) \leq f_{t^{\prime}}^{\prime}(x)$ at all damage levels $x \geq 0$, with $f_{t}^{\prime}(x)$ (resp. $f_{t^{\prime}}^{\prime}(x)$ ) naturally read as $\infty$ if $x$ is infeasible, i.e. if $x \notin \mathbf{X}_{t}$ (resp. $x \notin \mathbf{X}_{t^{\prime}}$ ).
} 
- the linear form $f_{t}(x)=t x$ (so $f_{t}^{\prime}(x)=t$ ), or more generally,

- the form $f_{t}(x)=t x^{c}$ for a fixed $c>0\left(\right.$ so $\left.f_{t}^{\prime}(x)=c t x^{c-1}\right)$,

because $f_{t}^{\prime}$ is then non-negative and increasing in $t$.

Theorem 1 Consider the unidimensional policy choice problem UP. Then, for every individual whose preferences are regular (i.e. satisfy R1-R3),

(a) the effect of toughness on terrorism is the sum of the deterrence and provocation effects: $E=P E+D E$;

(b) the two subeffects are competing, that is:

- $P E \geq 0$ if individual preferences satisfy NIP;

- $D E \leq 0$ if individual preferences satisfy TC and policies satisfy MP.

This theorem (proved in Appendix B) confirms Section 2's analysis of a trade-off between deterrence and provocation, this time from a marginal toughness angle, i.e. from a comparative statics angle. Indeed, under Theorem 1's conditions the two subeffects pull in opposite directions, and whether a marginal toughness rise increases terrorism by the person depends on which of $P E$ and $D E$ dominates. Arguably, this comparison is what policy makers should mainly focus on in practice.

Of course, Theorem 1 implies an analogous decomposition at the aggregate level: $\mathbf{E}=\mathbf{P E}+\mathbf{D E}$, with $\mathbf{E}, \mathbf{P E}$, and $\mathbf{P E}$ defined as the sum-total of the individual effects $E, P E$ and $D E$ across the population, respectively.

\subsection{The social utility of toughness}

So far I have been largely informal about the policy maker's preferences, occasionally assuming that he minimises sum-total terrorism or sum-total terrorism plus policy costs. More generally, assume now he holds some arbitrary preference order over the set $\mathbf{R}_{+}^{N} \times T$ of damages-policy combinations $\left(\left(x_{i}\right)_{i \in N}, t\right)$, and let this preference be representable by a 'social utility' function $U: \mathbf{R}_{+}^{N} \times T \rightarrow \mathbf{R}$ such that

- (terrorism-aversion) $U$ is an (at least weakly) decreasing function of each individual $i$ 's damage level $x_{i} \in \mathbf{R}_{+}$.

There are numerous examples. Social utility may be defined by $U\left(\left(x_{i}\right)_{i \in N}, t\right)=$ $-\sum_{i \in N} x_{i}$ if the policy maker minimises sum-total terrorism, or by $U\left(\left(x_{i}\right)_{i \in N}, t\right)=$ $\#\left\{i \in N: x_{i}=0\right\}$ if he minimises the number of terrorists (individuals with positive damage). A more general specification is $U\left(\left(x_{i}\right)_{i \in N}, t\right)=-\sum_{i \in N} x_{i}^{\alpha}$ (with a fixed parameter $\alpha>0$ ), which reduces to the first example if $a=1$ and to the second one if $\alpha \rightarrow 0$. Another natural class of utility functions are the Cobb-Douglas forms $U\left(\left(x_{i}\right)_{i \in N}, t\right)=\prod_{i \in N} x_{i}^{\alpha}$ (for some parameter $\alpha>0$ ). Each of these specifications can be refined by subtracting a (suitably scaled) cost term $\operatorname{Cost}(t)$ that captures financial costs or other negative policy effects 
such as loss of (civilian or military) lives or loss of life quality through more state supervision; this gives for instance the utility specification $U\left(\left(x_{i}\right)_{i \in N}, t\right)=$ $-\sum_{i \in N} x_{i}-\operatorname{Cost}(t)$. Note that by subtracting a cost term we assume that policy costs are additively separable from the damage disutility. A specification without additive separability is $U\left(\left(x_{i}\right)_{i \in N}, t\right)=t^{-\beta} \Pi_{i \in N} x_{i}^{\alpha}$ (for fixed parameters $\alpha, \beta>0)$, assuming here that $t$ is a toughness level taken from a policy interval $T \subseteq(0, \infty)$ and that toughness is costly (i.e. $\beta>0)$.

We now proceed to a comparative statics analysis that makes the following assumptions. As in the last two subsections, we consider the unidimensional policy choice problem UP, and assume that individual preferences on the damage-punishment quadrant $\mathbf{R}_{+}^{2}$ are regular (i.e. satisfy R1-R3), so that each individual $i$ has to any toughness level $t \in T$ a unique optimal damage response $\mathrm{x}_{i}(t)$, which is differentiable in $t$. Further, let the utility function $U$ be differentiable. Then the marginal value of toughness $t$ can be captured by the total derivative $d U / d t$ (evaluated at the status quo $\bar{t}$ ). By the chain rule,

$$
\underbrace{\frac{d U}{d t}}_{\begin{array}{c}
\text { marginal } \\
\text { utility of } \\
\text { toughness }
\end{array}}=\sum_{i \in N} \underbrace{\frac{\partial U}{\partial x_{i}}}_{\begin{array}{c}
\leq 0 \\
\text { marginal } \\
\text { utility of } i \text { 's } \\
\text { damage }
\end{array}} \underbrace{\frac{d \mathrm{x}_{i}}{d t}}_{\begin{array}{c}
\leq 0 \text { or } \geq 0 \\
\text { damage } \\
\text { response }
\end{array}}+\underbrace{\frac{\partial U}{\partial t}}_{\begin{array}{c}
\leq 0 \\
\text { cost } \\
\text { effect }
\end{array}} .
$$

So the marginal utility of toughness is composed of a (direct) cost effect $\frac{\partial U}{\partial t}$ and (indirect) effects $\frac{\partial U}{\partial x_{i}} \frac{d \mathrm{x}_{i}}{d t}(i \in N)$ through people's responses. While the cost effect is typically negative because toughness is expensive, the indirect effects can go in either direction because the sign of the damage response $\frac{d \mathrm{x}_{i}}{d t}$ may differ across individuals $i$. Using Theorem 1, we can decompose the damage response into the sum $\frac{d \mathrm{x}_{i}}{d t}=D E_{i}+P E_{i}$ of the deterrence effect $D E_{i}$ and the provocation effect $P E_{i}$ on individual $i$ 's damage, where typically $D E_{i} \leq 0$ and $P E_{i} \geq 0$. So, (2) becomes

$$
\underbrace{\frac{d U}{d t}}_{\begin{array}{c}
\text { marginal } \\
\text { utility of } \\
\text { toughness }
\end{array}}=\underbrace{\sum_{i \in N} \frac{\partial U}{\partial x_{i}} D E_{i}}_{\begin{array}{c}
\geq 0 \\
\text { aggregate deterrence } \\
\text { effect on utility }
\end{array}}+\underbrace{\sum_{i \in N} \frac{\partial U}{\partial x_{i}} P E_{i}}_{\begin{array}{c}
\leq 0 \\
\text { aggregate provocation } \\
\text { effect on utility }
\end{array}}+\underbrace{\frac{\partial U}{\partial t}}_{\begin{array}{c}
\leq 0 \\
\text { cost } \\
\text { effect }
\end{array}} .
$$

Three competing forces thus act on the marginal utility of toughness: $\frac{d U}{d t}$ is increased by an aggregate deterrence term, but decreased both by an aggregate provocation term and the cost effect. Whether a toughness increase is desirable depends on whether the deterrence term overweighs the two other terms.

Suppose further the policy maker does not care about who creates damage, in the sense that $U\left(\left(x_{i}\right)_{i \in N}, t\right)=U\left(\left(x_{i}^{\prime}\right)_{i \in N}, t\right)$ whenever the damage profiles $\left(x_{i}\right)_{i \in N}$ and $\left(x_{i}^{\prime}\right)_{i \in N}$ display identical total damage $\sum_{i \in N} x_{i}=\sum_{i \in N} x_{i}^{\prime}$. As 
one easily shows, the partial derivative $\frac{\partial U}{\partial x_{i}}$ is then the same for each individual $i$; so it can be bracketed out in (3), and we obtain

$$
\underbrace{\frac{d U}{d t}}_{\begin{array}{c}
\text { marginal } \\
\text { utility of } \\
\text { toughness }
\end{array}}=\underbrace{\frac{\partial U}{\partial x_{i}}}_{\begin{array}{c}
\leq 0 \\
\text { marginal } \\
\text { utility of } \\
\text { terrorism }
\end{array}}(\underbrace{\mathbf{D E}}_{\begin{array}{c}
\leq 0 \\
\text { efrence }
\end{array}}+\underbrace{\mathbf{P E}}_{\begin{array}{c}
\geq 0 \\
\text { aggregate } \\
\text { provocation } \\
\text { effect }
\end{array}})+\underbrace{\frac{\partial U}{\partial t}}_{\begin{array}{c}
\leq 0 \\
\text { cost } \\
\text { effect }
\end{array}},
$$

where $\mathbf{D E}$ and $\mathbf{P E}$ are, as usual, the aggregate deterrence effect $\sum_{i \in N} D E_{i}$ resp. provocation effect $\sum_{i \in N} P E_{i}$. Whether raising toughness is beneficial depends on the sign of (4). It is beneficial if (4) is positive, which (assuming that $\frac{\partial U}{\partial x_{i}}$ is strictly negative) happens exactly when

$$
\underbrace{\mathbf{D E}}_{\leq 0}+\underbrace{\mathbf{P E}}_{\geq 0}<\underbrace{-\frac{\partial U / \partial t}{\partial U / \partial x_{i}}}_{\leq 0},
$$

i.e. when the two competing effects, DE and PE, are overall 'sufficiently negative'. By contrast, a provocation-neglecting policy maker (who believes that preferences are policy-invariant, hence that $\mathbf{P E}=0$ ) raises toughness already if

$$
\mathbf{D E}<-\frac{\partial U / \partial t}{\partial U / \partial x_{i}}
$$

hence more easily because $\mathbf{P E} \geq 0$. The criteria (5) and (6) illustrate the behavioural difference between accounting for and neglecting provocation: in his decision over whether to raise toughness, the provocation-aware policy maker is guided by the more restrictive criterion (5), hence raises toughness less easily and reduces toughness more easily. The equilibrium toughness level (at which the policy maker neither raise nor reduces toughness) is lower for the provocation-aware policy maker, because his first-order condition

$$
\mathbf{D E}+\mathbf{P E}=-\frac{\partial U / \partial t}{\partial U / \partial x_{i}}
$$

is typically satisfied at a lower status quo toughness than the provocationneglecting policy maker's first-order condition

$$
\mathbf{D E}=-\frac{\partial U / \partial t}{\partial U / \partial x_{i}}
$$

The different equilibrium conditions (7) and (8) again illustrate the different toughness dispositions underlying provocation-aware and -neglecting policy making. 


\section{Concluding remarks}

First, let me summarise informally some points that have been developed formally. A policy maker may try to reduce terrorism either by cause-related measures, which aim to appease people's preferences, or by symptom-related measures, which change the constraints (feasible set) under which terrorists maximise their preference and which, importantly, may have side effects on some people's preference ('provocation'). While both approaches are costly, a fundamental difference lies in the benefit side: cause-related measures reduce terrorism (the only question being: by how much?), whereas symptomrelated measures may or may not reduce terrorism, depending on whether the deterrence effect overweighs the provocation effect. This does not imply that symptom-related measures are generally inferior, but that they bear a higher downside-risk: the worst outcome of a cause-related measure is to incur the policy cost without terrorism reduction, but the worst outcome of a symptomrelated measure (e.g. a war) is to incur the policy cost with a terrorism increase. Most of the paper has focussed on analysing symptom-related policies, and specifically the trade-off between deterrence and provocation. Theorem 1 provides general sufficient conditions under which the marginal deterrence effect is non-positive (i.e. terrorism-reducing) and the marginal provocation effect is non-negative (i.e. terrorism-increasing). I have argued that it is easy in practice to overlook provocation effects (Section 2.5). Provocation-neglect leads to toughness exaggeration (the fallacy of neglecting provocation), as argued informally in Section 2.5 and shown formally in Section 3.4 by comparing the (first-order) conditions under which a provocation-aware and a provocationneglecting policy maker chooses how tough to be. As an analytic example, Theorem 2 in Appendix A characterises optimal policies for a stylised objective function (minimising the number of terrorists), again confirming that there is a trade-off between deterring and provoking, and that provocation-neglect leads to toughness exaggeration.

Our analysis poses several empirical and theoretical challenges. On the empirical side, it would be of high practical interest to know the extent to which concrete cause-related policies (investments into social stability, into education etc.) appease preferences, ${ }^{18}$ and the extent to which concrete symptom-related policies (weapons embargoes, military presence, criminal legislation etc.) brutalise preferences, i.e. provoke. Among different ways to deter (i.e. to render the feasible set $\mathbf{F} \subseteq \mathbf{R}_{+}^{2}$ 'steeper'), which ones provoke least? Are there policies that deter without provoking? A concrete hypothesis to investigate is whether defensive deterrence provokes less than aggressive deterrence. As mentioned in Section 2.2, I count a deterrence measure as defensive if it makes it harder to create terrorism (e.g. weapons embargoes) and as aggressive if it increases

\footnotetext{
${ }^{18}$ This question relates to existing empirical research apart from the difference between appeasing preferences and reducing terrorism.
} 
punishment (e.g. tough criminal legislation). ${ }^{19}$ To provide answers to such questions, it would help to understand whether and when provocability is a form of reciprocity, or of taste acquisition, or of dynamic inconsistency, a psychological question (see Section 2.4).

On a theoretical dimension, there is plenty of room for adapting our model to concrete applications, or for 'merging' it with models studied in political economy (that is, incorporating provocation into these models), or for refining the strategic interaction between individuals and the policy maker. For instance, one might introduce uncertainty of the policy maker about the types of population members, i.e., about how brutal and how provocable their preferences are. Or, one might model a repeated interaction between policy maker and population, with the question arising as to whether toughness can provoke only in the short run or can have lasting provocation effects on the population.

\section{References}

Becker, Gary. 1996. Accounting For Tastes. Harward University Press, Cambridge Mass., London.

Bénabou, Roland and Marek Pycia. 2002. "Dynamic inconsistency and self-control: a planner-doer interpretation." Economic Letters, 77: 419-424.

Bolton, Gary E. and Axel Ockenfels. 2000. "ERC: a theory of equity, reciprocity and competition." American Economic Review, 90: 166-193.

Bowles, Samuel. 1998. "Endogenous preferences: the cultural consequences of markets and other economic institutions." Journal of Economic Literature, 36(1): 75-111.

Cioffi-Revilla, Claudio. 1998. "On the likely magnitude, extent, and duration of an Iraq-UN war." Journal of Conflict Resolution, 35: 387-411.

Cioffi-Revilla, Claudio. 1985. "Political reliability theory and war in the International system." American Journal of Political Science, 29: 47-68.

Dekel, Eddie, Jeff Ely and Okan Yilankaya. 2007. "Evolution of Preferences." The Review of Economic Studies, 74: 685-704.

Dufwenberg, Martin and Georg Kirchsteiger. 2004. "A theory of sequential reciprocity." Games and Economic Behavior, 47(2): 268-298.

\footnotetext{
${ }^{19}$ Both kinds of deterrence can be defined formally and do indeed constitute deterrence, i.e. render the feasible set $\mathbf{F} \subseteq \mathbf{R}_{+}^{2}$ 'steeper'. Let $A$ be a policy-independent set of possible (modes of) behaviour, and let $\mathrm{x}(a, t) \in \mathbf{R}_{+}$resp. $\mathrm{y}(a, t) \in \mathbf{R}_{+}$be the damage resp. punishment resulting from behaviour $a \in A$ at policy $t \in T$. So policy $t \in T$ leads to the feasible set $\mathbf{F}_{t}=\{(\mathrm{x}(a, t), \mathrm{y}(a, t)): a \in A\} \subseteq \mathbf{R}_{+}^{2}$. Say that one policy does more defensive (resp. aggressive) deterrence than another if it implies lower damage $\mathrm{x}(a, t)$ (resp. higher punishment $\mathrm{y}(a, t))$ for each individual behaviour $a \in A$. Installing a (functioning) missile defense system, for instance, constitutes defensive deterrence: the action $a$ of sending a missile would not anymore create damage.
} 
Dumas, Lloyd. 2002. "Is development an effective way to fight terrorism?" Philosophy \& Public Policy Quarterly, 22(4): 7-12.

Enders, Walter and Todd Sandler. 2006. The political Economy of Terrorism. Cambridge University Press.

Eubank, William and Leonard Weinberg. 1994. "Does democracy encourage terrorism?" Terrorism and Political Violence, 6(4): 155-64.

Falk, Armin and Urs Fischbacher. 2006. "A Theory of Reciprocity." Games and Economic Behavior, 54 (2): 293-315.

Fehr, Ernst and Simon Gächter. 1998. "Reciprocity and economics: the economic implications of homo reciprocans." European Economic Review, 42: 845-859.

Frey, Bruno. 2004. Dealing with Terrorism: Stick or Carrot? Edward Elgar Publishing Ltd., Cheltenham, UK and Northampton, Mass.

Hammond, Peter. 1976. "Changing Tastes and Coherent Dynamic Choice." Review of Economic Studies, 43: 159-173.

Hansson, Sven Ove. 1995. "Changes in preference." Theory and Decision, 38: $1-28$.

Jehle, Geoffrey and Philip Reny. 2001. Advanced Microeconomic Theory. 2nd Edition. International Edition.

O'Donoghue, Edward and Matthew Rabin. 1999. "Doing it now or doing it later." American Economic Review, 89: 103-124.

Polak, Robert. 1976. "Interdependent preferences." American Economic Review 66(3): 309-20.

Rabin, Matthew. 1993. "Incorporating fairness into game theory and economics." American Economic Review, 83: 1281-1302.

Rabin, Matthew. 1998. "Psychology and economics." Journal of Economic Literature, 36(1): 11-46.

Sethi, Rajiv and E. Somanathan. 2001. "Preference evolution and reciprocity." Journal of Economic Theory, 97: 273-297.

Sethi, Rajiv and E. Somanathan. 2003. "Understanding Reciprocity." Journal of Economic Behavior and Organization, 50(1): 1-27.

Silke, Andrew. 1994. Research on Terrorism: Trends, Achievements and Failures. Frank Cass Publishers, New York.

Simon, Carl and Lawrence Blume. 1994. Mathematics for Economists. Norton \& Co. Inc., New York.

Strotz, Robert H. 1955-56. "Myopia and inconsistency in dynamic utility maximization." Review of Economic Studies, 23(3): 165-180.

Tonry, Michael. 1998. Handbook on Crime and Punishment. Oxford University Press. 


\section{A An example: minimising the number of ter- rorists}

Unlike in much of this paper, policies may now contain both cause- and symptomrelated measures; they might be represented as vectors $t=\left(t_{1}, \ldots, t_{k}\right)$ of positions on $k$ cause- or symptom-related dimensions. While I have so far ensured high generality of preferences by placing no specific restrictions (except from plausible ones such as punishment-aversion of individuals and terrorism-aversion of the policy maker), let us now turn to concrete preferences. I take the policy maker to follow a paradigmatic and simple objective - minimising the number of terrorists - and the individuals to hold preferences from a plausible but special class. This will allow us to analytically determine optimal policies. The upshot will be that optimal policies again have to strike the right compromise between provocation and deterrence, albeit in a particular sense.

Specifically, individual preferences fall into the following class.

Preference Model PM. Each individual in the population $N$ is of one of the following types.

Either he has a peaceful type, meaning that under each policy $t \in T$ his preference order $\succeq_{t}$ (on $\mathbf{R}_{+}^{2}$ ) is peaceful as defined Section 2.1. (The set of peaceful types can be defined as the set of families $\left(\succeq_{t}\right)_{t \in T}$ of peaceful preference orders.)

Or his type belongs to the set $\mathbf{R}$ of possibly brutal types. Each possibly brutal type $\theta$ in $\mathbf{R}$ ( $\theta$ represents the status quo damage-inclination) holds under every policy $t \in T$ a preference order $\succeq_{t} \equiv \succeq_{t, \theta}$ that is punishment-averse (see Section 2.1), strictly convex ${ }^{20}$, and representable by a utility function $u_{t}: \mathbf{R}_{+}^{2} \rightarrow$ $\mathbf{R}$ of the (separable) form

$$
u_{t}(x, y)=v_{t}(x)-d(y)(\text { for all } x, y \geq 0)
$$

with the following interpretation and properties:

- $d(y)$ represents disutility from punishment, where the function $d: \mathbf{R}_{+} \rightarrow$ $\mathbf{R}$ is policy-independent (a plausible restriction) and differentiable.

- $v_{t}(x)$ represents utility of damage and takes the form $v_{t}(x)=v(x-\theta-$ $P(t)$ ), where:

$-v: \mathbf{R} \rightarrow \mathbf{R}$ is a differentiable and strictly concave function that peaks at 0 ; hence $v_{t}(x)$ peaks at $x=\theta+P(t)$, and so the type has a brutal preference with preferred damage level $\theta+P(t)$ under policies

\footnotetext{
${ }^{20}$ That is, for every $(x, y) \in \mathbf{R}_{+}^{2}$ the upper contour set $\left\{\left(x^{\prime}, y^{\prime}\right) \in \mathbf{R}_{+}^{2}:\left(x^{\prime}, y^{\prime}\right) \succeq_{t}(x, y)\right\}$ is strictly convex. It follows that $u_{t}$ is quasi-concave (Th. A1.14 in Geoffrey Jehle and Philip Reny 2001; to be precise, this theorem uses a slightly stronger notion of strictly convex preferences, which is implied by ours given that we also assume $\succeq_{t}$ to be continuous and punishment-averse).
} 
$t$ with $\theta+P(t)>0$, and peaceful preferences under policies $t$ with $\theta+P(t) \leq 0$.

- $P(t) \in \mathbf{R}$ is interpreted as the amount by which policy $t$ provokes ${ }^{21}$, i.e. increases the preferred damage level from the status quo $\bar{t}$, and accordingly I assume without loss of generality that $P(\bar{t})=0{ }^{22}$

$-\theta$ is interpreted as the status quo damage-inclination, as it is the status quo preferred damage level (by $P(\bar{t})=0$ ) provided $\theta \geq 0$.

- $v, d$ and $P(t)$ are the same across types $\theta \in \mathbf{R}$ (this is the main restriction, essential for analytic tractability).

- There is no policy $t \in T$ at which all types $\theta \in \mathbf{R}$ most prefer the nodamage-no-punishment pair $(x, y)=(0,0)$ from $\mathbf{F}_{t}$ (this excludes trivial solutions to the problem of minimising the number of terrorists).

PM is a flexible model: the precise forms of $v, d, P, T$ can be chosen to match the intended application. As as simple example, let the policy space be unidimensional, say the interval $T=\mathbf{R}_{+}$of toughness levels, let disutility of punishment be linear, i.e. given by $d(y)=b y$ for a fixed parameter $b>0$, let provocation be also linear, i.e. given by $P(t)=(t-\bar{t}) p$ for a fixed parameter $p>$ 0 (recall that $P(t)$ represents the change of preferred damage level if toughness changes from the status quo level $\bar{t}$ to $t$ ), and let the function $v$ be given by $v(x):=-|x|^{a}$ for a fixed parameter $a>2$. In summary, then, under toughness $t \in \mathbf{R}_{+}$type $\theta \in \mathbf{R}$ has the (quasi-linear) utility function

$$
u_{t}(x, y)=-|x-\theta-(t-\bar{t}) p|^{a}-b y \text { for all }(x, y) \in \mathbf{R}_{+}^{2},
$$

with preferred damage level given by $\theta+(t-\bar{t}) p$ (or by 0 if this number is $<0$ ).

While the policy space $T$ is arbitrary (perhaps multi-dimensional with causeand symptom-related dimensions), a convexity property is required:

Convex Punishment CP. For each policy $t \in T$, the feasible set $\mathbf{F}_{t}$ contains the no-damage-no-punishment pair $(0,0)$, it is (topologically) closed and connected, and its punishment function $f_{t}: \mathbf{X}_{t} \rightarrow \mathbf{R}_{+}$is weakly convex.

Recall that $f_{t}(x)$ represents the minimal punishment for damage $x \in \mathbf{X}_{t}$; the graph of $f_{t}$ is the southern border of the feasible set $\mathbf{F}_{t}$. By $\mathrm{CP}$, this southern border has a weakly convex shape, for instance a linear shape given by $f_{t}(x)=\alpha_{t} x$ for some policy-dependent slope $\alpha_{t} \geq 0$. In general, as $\mathbf{F}_{t}$ is connected and contains $(0,0)$, the feasible damage set $\mathbf{X}_{t} \subseteq \mathbf{R}_{+}$is an interval containing 0 , hence is either $\mathbf{R}_{+}$(unlimited feasibility) or of the form $\left[0, x_{t}^{*}\right]$ or $\left[0, x_{t}^{*}\right)$ (with a finite feasibility bound $x_{t}^{*}$ ).

\footnotetext{
${ }^{21}$ The more general term "affects preference" is perhaps better here than "provokes", as $t$ could contain cause-related measures (aimed at preference appeasement, i.e. at $P(t)<0$ ).

${ }^{22}$ One may always achieve $P(\bar{t})=0$ by subtracting $P(\bar{t})$ from each $P(t), t \in T$, while adding $P(\bar{t})$ to each individual's type $\theta \in \mathbf{R}$; this normalisation leaves individual preferences unchanged.
} 
Theorem 2 Assuming the preference model PM and convex punishment $C P$,

(a) the expression $D(t):=v^{\prime-1}\left(d^{\prime}(0) f_{t}^{\prime}(0)\right)$ is for each policy $t \in T$ well-defined, i.e. the (right hand) derivative $f_{t}^{\prime}(0)$ exists and $d^{\prime}(0) f_{t}^{\prime}(0)$ has a unique inverse image under the derivative function $v^{\prime}: \mathbf{R} \rightarrow \mathbf{R}$;

(b) (provocation-aware policies) each policy $t \in T$ that minimises $P(t)+D(t)$ minimises the number of terrorists if each individual's response to each policy $t \in T$ maximises his preference within the feasible set $\mathbf{F}_{t}$;

(c) (provocation-neglecting policies) each policy $t \in T$ that minimises $D(t)$ minimises the number of terrorists if each individual's response to each policy $t \in T$ maximises his status quo preference within the feasible set $\mathbf{F}_{t}$

(d) the individual responses assumed in (b) and (c) exist and are unique.

Taking the example given after the definition of PM and assuming each toughness level $t \in T=\mathbf{R}_{+}$leads to the (maximal) feasible damage set $\mathbf{X}_{t}=$ $\mathbf{R}_{+}$and to a linear punishment function given by $f_{t}(x)=t x$ (which becomes 'steeper' if toughness $t$ increases), one finds that $D(t)=-(t b / a)^{1 /(a-1)}$, and by minimising $P(t)+D(t)=(t-\bar{t}) p-(t b / a)^{1 /(a-1)}$ one finds the

$$
\text { optimal toughness level: } t=\frac{(b / a)^{1 /(a-2)}}{(p(a-1))^{(a-1) /(a-2)}}
$$

(as derived at the end of Appendix B). So optimal toughness increases if marginal provocation $p=P^{\prime}(t)$ falls (i.e. if toughness provokes less), and also if marginal punishment aversion $b=d^{\prime}(y)$ increases (i.e. if punishment hurts more, hence deters more). These comparative statics confirm our intuition.

Theorem 2 once again confirms the trade-off between provocation and deterrence: $P(t)$ is a measure for how much the policy $t$ provokes, and $D(t)$ is an (inverse) measure for how much $t$ deters. $D(t)$ measures deterrence in that it reflects the punishment function $f_{t}$ but not any policy-induced preference change. $D(t)$ measures deterrence inversely: the more deterring $t$, the 'steeper' the feasible set $\mathbf{F}_{t}$, hence the higher the derivative $f_{t}^{\prime}(0)$, so the higher the product $d^{\prime}(0) f_{t}^{\prime}(0)$, and therefore, the lower $D(t)=v^{\prime-1}\left(d^{\prime}(0) f_{t}^{\prime}(0)\right)$ because we have applied a strictly decreasing function $v^{\prime-1} .^{23}$

Figure 10 illustrates the (one-dimensional) case that $t$ is a toughness level from a toughness interval $T \subseteq \mathbf{R}$. Plausibly, the higher toughness $t$, the larger $P(t)$ (more provocation) and the smaller $D(t)$ (more deterrence), the goal being to minimise the sum $P(t)+D(t)$. If $T$ is multidimensional, the trade-off becomes multi-dimensional, possibly with cause-related dimensions.

By contrast, the provocation-neglecting policy maker believes that $P(t)=$ 0 (no provocation) for all (arbitrarily tough) policies $t \in T$, hence what he minimises is not $P(t)+D(t)$ but $D(t)$, leading to toughness exaggeration.

\footnotetext{
${ }^{23} v^{\prime}: \mathbf{R} \rightarrow \mathbf{R}$ is a strictly decreasing function, so has strictly decreasing inverse function $v^{\prime-1}: v(\mathbf{R}) \rightarrow \mathbf{R}$.
} 


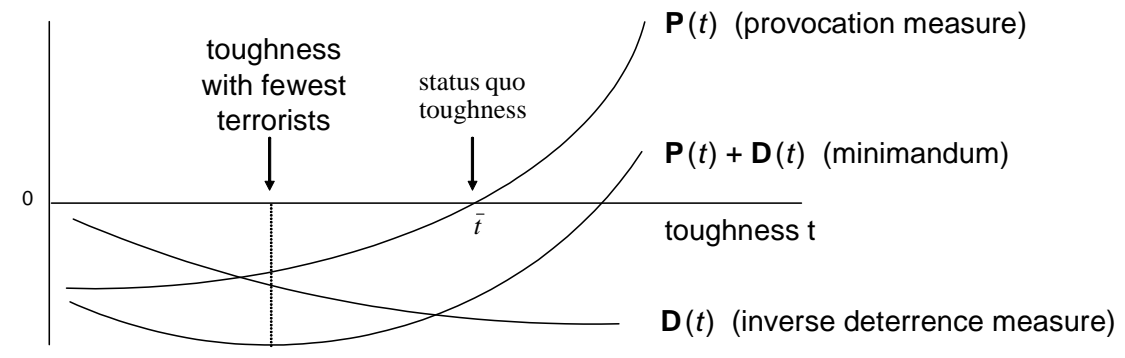

Figure 10: The trade-off in Theorem 2 for a unidimensional policy space $T$

Perhaps surprisingly, the optimal policies in Theorem 2 do not depend on the distribution of types across the population: minimising $P(t)+D(t)$ is optimal regardless of how many individuals are highly damage-inclined (large $\theta \in \mathbf{R}$ ). So the policy maker can set its policy without 'understanding' people. This interesting feature of the model (with its stylised notion of optimality: minimising the number of terrorists) is certainly an exception. In other models, optimal policies are type-distribution-sensitive and often analytically intractable.

Finally, the provocation and deterrence measures $P(t)$ and $D(t)$ differ from the earlier-studied provocation and deterrence effects $\mathbf{P E}(t)$ and $\mathbf{D E}(t)$ because they arise in the context of minimising the number of terrorists, not the sumtotal amount of terrorism as earlier.

\section{B Proof of Theorems 1 and 2}

Proof of Theorem 1. Suppose UP and R1-R3.

(a) By $\mathrm{x}(t)=\mathrm{x}(t, t)$, the function $t \mapsto \mathrm{x}(t)$ is the composition of the differentiable functions $t \mapsto(t, t)$ (from $T$ to $T \times T$ ) and the by R3 differentiable function $\left(t_{1}, t_{2}\right) \mapsto \mathrm{x}\left(t_{1}, t_{2}\right)$ (from $T \times T$ to $\left.\mathbf{R}_{+}\right)$. Hence, by the chain rule, $t \mapsto \mathrm{x}(t)$ is itself differentiable and

$$
\mathrm{x}^{\prime}(t)=\frac{\partial}{\partial t_{1}} \mathrm{x}(t, t)+\frac{\partial}{\partial t_{2}} \mathrm{x}(t, t) \text { at all } t \in T .
$$

Setting $t=\bar{t}$, the left-hand side becomes $E$, and the right-hand side is recognised as the sum of $P E$ and $D E$; for instance,

$$
\frac{\partial}{\partial t_{1}} \mathrm{x}(\bar{t}, \bar{t})=\left.\frac{\partial}{\partial t_{1}} \mathrm{x}(t, \bar{t})\right|_{t=\bar{t}}=\left.\frac{d}{d t} \mathrm{x}_{\mathrm{prov}}(t)\right|_{t=\bar{t}}=P E .
$$

(b) Throughout, I write $(\bar{x}, \bar{y})$ for the pair $(\mathrm{x}(\bar{t}, \bar{t}), \mathrm{y}(\bar{t}, \bar{t}))\left(=\left(\mathrm{x}_{\text {prov }}(\bar{t}), \mathrm{y}_{\text {prov }}(\bar{t})\right)=\right.$ $\left.\left(\mathrm{x}_{\text {deter }}(\bar{t}), \mathrm{y}_{\text {deter }}(\bar{t})\right)\right)$. 
1. In this part I assume NIP and show that $P E \geq 0$. Suppose for a contradiction that $P E<0$. I establish several claims; the last one contains the desired contradiction.

Claim 1. There exists a toughness level $\tilde{t} \in T$ larger than $\bar{t}$ such that $\mathrm{x}_{\text {prov }}(t)<\bar{x}$ for all $t \in(\bar{t}, \tilde{t}]$. I write $\tilde{x}:=\mathrm{x}_{\text {prov }}(\tilde{t})$ and $\tilde{y}:=\mathrm{y}_{\text {prov }}(\tilde{t})$.

As $P E=\lim _{t \rightarrow \bar{t}} \frac{\mathrm{x}_{\text {prov }}(t)-\mathrm{x}_{\text {prov }}(\bar{t})}{t-\bar{t}}=\lim _{t \rightarrow \bar{t}} \frac{\mathrm{x}_{\text {prov }}(t)-\bar{x}}{t-\bar{t}}$ and as $P E<0$, we have $\frac{\mathrm{x}_{\text {prov }}(t)-\bar{x}}{t-\bar{t}}<0$ for all $t \neq \bar{t}$ in a sufficiently small neighbourhood of $\bar{t}$. In particular, there is a $\tilde{t}>\bar{t}$ such that for all $t \in(\bar{t}, \tilde{t}]$ we have $\mathrm{x}_{\text {prov }}(t)-\bar{x}<0$, i.e. $\mathrm{x}_{\text {prov }}(t)<\bar{x}$, q.e.d.

Claim 2. For every damage level $x \in[\tilde{x}, \bar{x}]$ there exists a toughness level $t \in[\bar{t}, \tilde{t}]$ such that $\mathrm{x}_{\text {prov }}(t)=x$.

Let $x \in[\tilde{x}, \bar{x}]$. As the function $\mathrm{x}_{\text {prov }}$ is continuous on $[\bar{t}, \tilde{t}]$ (because it is differentiable) and as $\mathrm{x}_{\text {prov }}(\tilde{t})=\tilde{x} \leq x \leq \bar{x}=\mathrm{x}_{\text {prov }}(\bar{t})$, the intermediate value theorem implies the existence of a $t \in[\bar{t}, \tilde{t}]$ such that $\mathrm{x}_{\text {prov }}(t)=x$, q.e.d.

For every damage level $x \in[\tilde{x}, \bar{x}]$, define

$$
S(x):=\sup \left\{y \geq 0: u_{\bar{t}}(x, y) \geq u_{\bar{t}}(\tilde{x}, \tilde{y})\right\}\left(\in \mathbf{R}_{+} \cup\{\infty,-\infty\}\right),
$$

with the usual conventions that $\sup \emptyset:=-\infty$ and that $\sup Q:=\infty$ whenever $Q \subseteq \mathbf{R}$ has no upper bound.

Claim 3. $S(\tilde{x})=\tilde{y}$.

By definition of $S(\tilde{x})$ we have $S(\tilde{x}) \geq \tilde{y}$, and using punishment-aversion it follows that $S(\tilde{x})=\tilde{y}$, q.e.d.

Claim 4. For all $x \in[\tilde{x}, \bar{x}]$, if $S(x) \in \mathbf{R}_{+}$then $u_{\bar{t}}(x, S(x))=u_{\bar{t}}(\tilde{x}, \tilde{y})$.

Consider any $x \in[\tilde{x}, \bar{x}]$ with $S(x) \in \mathbf{R}_{+}$. To show that $u_{\bar{t}}(x, S(x)) \geq$ $u_{\bar{t}}(\tilde{x}, \tilde{y})$, note that by definition of $S(x)$ there is a sequence $\left(y_{k}\right)_{k=1,2, \ldots}$ in $[0, S(s)]$ converging to $S(x)$ such that $u_{\bar{t}}\left(x, y_{k}\right) \geq u_{\bar{t}}(\tilde{x}, \tilde{y})$ for all $k=1,2, \ldots$ As $u_{\bar{t}}$ is a continuous function, $u_{\bar{t}}\left(x, y_{k}\right) \rightarrow u_{\bar{t}}(x, S(x))$ as $k \rightarrow \infty$. So, as weak inequalities are preserved in the limit, $u_{\bar{t}}(x, S(x)) \geq u_{\bar{t}}(\tilde{x}, \tilde{y})$. To show the converse inequality, consider any sequence $\left(z_{k}\right)_{k=1,2, \ldots}$ in $(S(x), \infty)$ converging to $S(x)$ (of course there is one). By definition of $S(x)$, we have $u_{\bar{t}}\left(x, z_{k}\right)<u_{\bar{t}}(\tilde{x}, \tilde{y})$ for all $k=1,2, \ldots$ So, again by continuity of $u_{\bar{t}}, u_{\bar{t}}(x, S(x)) \leq u_{\bar{t}}(\tilde{x}, \tilde{y})$, q.e.d.

Claim 5. $S(\bar{x})=\infty$.

For a contradiction, suppose $S(\bar{x}) \neq \infty$. We also have $S(\bar{x}) \neq-\infty$ because $u_{\bar{t}}(\bar{x}, \bar{y}) \geq u_{\bar{t}}(\tilde{x}, \tilde{y})\left(\right.$ as $(\bar{x}, \bar{y})$ maximises $u_{\bar{t}}(x, y)$ subject to $\left.(x, y) \in \mathbf{F}_{\bar{t}}\right)$. So $S(\bar{x}) \in \mathbf{R}_{+}$. Hence, by Claim 4 ,

$\left.{ }^{*}\right) u_{\bar{t}}(\bar{x}, S(\bar{x}))=u_{\bar{t}}(\tilde{x}, \tilde{y})$.

This and the inequality $u_{\bar{t}}(\bar{x}, \bar{y})>u_{\bar{t}}(\tilde{x}, \tilde{y})$ (which holds because $(\bar{x}, \bar{y})$ uniquely maximises $u_{\bar{t}}(x, y)$ subject to $\left.(x, y) \in \mathbf{F}_{\bar{t}}\right)$ imply that $u_{\bar{t}}(\bar{x}, \bar{y})>$ $u_{\bar{t}}(\bar{x}, S(\bar{x}))$, which by punishment-aversion entails that

$(* *) \bar{y}<S(\bar{x})$. 
But $\left(^{*}\right)$ also implies that $u_{\tilde{t}}(\tilde{x}, \tilde{y}) \leq u_{\tilde{t}}(\bar{x}, S(\bar{x}))$ by NIP. Using $(* *)$ and punishment-aversion, it follows that $u_{\tilde{t}}(\tilde{x}, \tilde{y})<u_{\tilde{t}}(\bar{x}, \bar{y})$, a contradiction since $(\tilde{x}, \tilde{y})$ maximises $u_{\tilde{t}}(x, y)$ subject to $(x, y) \in \mathbf{F}_{\bar{t}}$, q.e.d.

Claim 6. There exists an $x \in[\tilde{x}, \bar{x}]$ that is smallest subject to $S(x)=\infty$. I denote it by $x_{\infty}$.

I have to show that the set $X_{\infty}:=\{x \in[\tilde{x}, \bar{x}]: S(x)=\infty\}$ has a smallest element. By Claim 5, $X_{\infty}$ is non-empty. So it has an infimum $x_{*}:=\inf X_{\infty}$ in $[\tilde{x}, \bar{x}]$. I have to show that $S\left(x_{*}\right)=\infty$ (i.e. that the infimum is a minimum). By definition of $x_{*}$, there is a sequence $\left(x_{k}\right)_{k=1,2, \ldots}$ in $X_{\infty}$ that converges to $x_{*}$. Consider any $y \geq 0$. As $u_{\bar{t}}$ is a continuous function, $u_{\bar{t}}\left(x_{k}, y\right) \rightarrow u_{\bar{t}}\left(x_{*}, y\right)$ as $k \rightarrow \infty$. Note also that, for all $k, u_{\bar{t}}\left(x_{k}, y\right) \geq u_{\bar{t}}(\tilde{x}, \tilde{y})$ : otherwise $u_{\bar{t}}\left(x_{k}, y^{\prime}\right)<$ $u_{\bar{t}}(\tilde{x}, \tilde{y})$ for all $y^{\prime}>y$ by punishment-aversion, implying that $S\left(x_{k}\right) \leq y$, in contradiction with $x_{k} \in X_{\infty}$. So, as weak inequalities are preserved in the limit, we have $u_{\bar{t}}\left(x_{*}, y\right) \geq u_{\bar{t}}(\tilde{x}, \tilde{y})$. Since this has been shown for all $y \geq 0$, we have $S\left(x_{*}\right)=\infty$, q.e.d.

Claim \%. $x_{\infty}>\tilde{x}$.

By Claim 3, $S(\tilde{x})=\tilde{y}$. So $S(\tilde{x})<\infty$. Hence $x_{\infty} \neq \tilde{x}$ by Claim 6, q.e.d.

Claim 8. $S(x) \rightarrow \infty$ as $x \uparrow x_{\infty}$.

Consider any sequence $\left(x_{k}\right)_{k=1,2, \ldots}$ in $\left[\tilde{x}, x_{\infty}\right)$ such that $x_{k} \uparrow x_{\infty}$. I have to show that $S\left(x_{k}\right) \rightarrow \infty$. For a contradiction, suppose that $S\left(x_{k}\right) \nrightarrow \infty$. Then there is a $\hat{y}>0$ and a subsequence $\left(x_{k_{j}}\right)_{j=1,2, \ldots}-$ I denote it simply by $\left(x_{j}^{\prime}\right)_{j=1,2, \ldots}$ - such that $S\left(x_{j}^{\prime}\right)<\hat{y}$ for all $j$. So, by definition of $S\left(x_{j}^{\prime}\right)$, we have $u_{\bar{t}}\left(x_{j}^{\prime}, \hat{y}\right)<u_{\bar{t}}(\tilde{x}, \tilde{y})$ for all $j$. Hence, as $u_{\bar{t}}\left(x_{j}^{\prime}, \hat{y}\right) \rightarrow u_{\bar{t}}\left(x_{\infty}, \hat{y}\right)$ by continuity of $u_{\bar{t}}$ and as weak inequalities are preserved in the limit, we have $u_{\bar{t}}\left(x_{\infty}, \hat{y}\right) \leq u_{\bar{t}}(\tilde{x}, \tilde{y})$. Hence, by punishment-aversion, we have $u_{\bar{t}}\left(x_{\infty}, y\right)<u_{\bar{t}}(\tilde{x}, \tilde{y})$ for all $y>\hat{y}$. So $S\left(x_{\infty}\right) \leq \hat{y}$, a contradiction since $S\left(x_{\infty}\right)=\infty$ by Claim 6 , q.e.d.

Claim 9. For all $y \geq 0$, we have $u_{\tilde{t}}\left(x_{\infty}, y\right) \geq u_{\tilde{t}}(\tilde{x}, \tilde{y})$.

Let $y \geq 0$. By Claim 8 there is an $x^{\prime} \in\left[\tilde{x}, x_{\infty}\right)$ such that $S(x) \geq y$ for all $x \in\left(x^{\prime}, x_{\infty}\right)$. For any $x \in\left(x^{\prime}, x_{\infty}\right)$, we have

- $u_{\tilde{t}}(x, y) \geq u_{\tilde{t}}(x, S(x))$ by punishment-aversion, and

- $u_{\tilde{t}}(x, S(x)) \geq u_{\tilde{t}}(\tilde{x}, \tilde{y})$ by NIP and the fact that $u_{\bar{t}}(x, S(x))=u_{\bar{t}}(\tilde{x}, \tilde{y})$ given Claim 4.

So $u_{\tilde{t}}(x, y) \geq u_{\tilde{t}}(\tilde{x}, \tilde{y})$ for all $x \in\left(x^{\prime}, x_{\infty}\right)$. Hence, as $u_{\tilde{t}}$ is continuous, $u_{\tilde{t}}\left(x_{\infty}, y\right) \geq u_{\tilde{t}}(\tilde{x}, \tilde{y})$, q.e.d.

Claim 10. $(\tilde{x}, \tilde{y})$ does not maximise $u_{\tilde{t}}(x, y)$ subject to $(x, y) \in \mathbf{F}_{\bar{t}}$ (a contradiction, completing the proof).

By Claim 2 there is a toughness level $t \in[\bar{t}, \tilde{t}]$ such that $\mathrm{x}_{\text {prov }}(t)=x_{\infty}$. Write $y_{\infty}:=\mathrm{y}_{\text {prov }}(t)$. I now apply Claim 9 to $y_{\infty}+1$, which gives us $u_{\tilde{t}}\left(x_{\infty}, y_{\infty}+1\right) \geq$ $u_{\tilde{t}}(\tilde{x}, \tilde{y})$. So, $u_{\tilde{t}}\left(x_{\infty}, y_{\infty}\right)>u_{\tilde{t}}(\tilde{x}, \tilde{y})$ by punishment-aversion. As $\left(x_{\infty}, y_{\infty}\right)=$ $\left(\mathrm{x}_{\text {prov }}(t), \mathrm{y}_{\text {prov }}(t)\right) \in \mathbf{F}_{\bar{t}}$, it follows that $(\tilde{x}, \tilde{y})$ does not maximise $u_{\tilde{t}}(x, y)$ subject to $(x, y) \in \mathbf{F}_{\bar{t}}$, q.e.d. 
2. I now assume TC and MP, and show that $D E \leq 0$. Suppose that $D E>0$. I derive a contradiction, again in several steps.

Claim 1. The feasible damage sets $\mathbf{X}_{t} \subseteq \mathbf{R}_{+}, t \in T$, are intervals containing 0 , and the function $t \mapsto \mathbf{X}_{t}$ is weakly decreasing (w.r.t. set-inclusion).

Each $\mathbf{X}_{t}$ is an interval containing 0 because it is the projection on the $x$ coordinate of the set $\mathbf{F}_{t} \subseteq \mathbf{R}_{+}^{2}$, which by MP is connected and contains $(0,0)$. Now consider $t<t^{\prime}$ in $T$. To show that $\mathbf{X}_{t^{\prime}} \subseteq \mathbf{X}_{t}$, let $x \in \mathbf{X}_{t^{\prime}}$. By the increasingness assumption in $\mathrm{MP}, f_{t}^{\prime}(x) \leq f_{t^{\prime}}^{\prime}(x)$; so $f_{t}^{\prime}(x)<\infty$, and hence $x \in \mathbf{X}_{t^{\prime}}$, q.e.d.

Claim 2. There exists a toughness level $\tilde{t} \in T$ larger than $\bar{t}$ such that $\mathrm{x}_{\text {deter }}(\tilde{t})>\bar{x}$. I henceforth write $\tilde{x}$ for $\mathrm{x}_{\text {deter }}(\tilde{t})$.

By assumption $D E=\mathrm{x}_{\text {deter }}^{\prime}(\bar{t})>0$, i.e. $\lim _{t \rightarrow \bar{t}} \frac{\mathrm{x}_{\text {deter }}(t)-\mathrm{x}_{\text {deter }}(\bar{t})}{t-\bar{t}}=\lim _{t \rightarrow \bar{t}} \frac{\mathrm{x}_{\text {deter }}(t)-\bar{x}}{t-\bar{t}}>$ 0 . So $\frac{\mathrm{x}_{\text {deter }}(t)-\bar{x}}{t-\bar{t}}>0$ for all $t \neq \bar{t}$ sufficiently close $\bar{t}$. Hence $\mathrm{x}_{\text {deter }}(t)-\bar{x}>0$ for all $t>\bar{t}$ sufficiently close $\bar{t}$, q.e.d.

Claim 3. $f_{t}(x)$ is (at least weakly) increasing in each argument, i.e. in $x \in \mathbf{X}_{t}$ (for each $t \in T$ ) and in $t \in\left\{t^{\prime} \in T: x \in \mathbf{X}_{t^{\prime}}\right\}$ (for each $x \geq 0$ ).

At any fixed $t \in T$, increasingness in $x \in \mathbf{X}_{t}$ holds since, by MP, $f_{t}^{\prime}(x) \geq 0$ at all $x \in \mathbf{X}_{t}$. To show increasingness in $t \in T$, consider a fixed $\hat{x} \geq 0$, and let $t_{-}, t_{+} \in\left\{t \in T: \hat{x} \in \mathbf{X}_{t}\right\}$ satisfy $t_{-} \leq t_{+}$. To show that $f_{t_{-}}(\hat{x}) \leq f_{t_{+}}(\hat{x})$, I define the function $g:[0, \hat{x}] \rightarrow \mathbf{R}$ by $g(x):=f_{t_{+}}(x)-f_{t_{-}}(x)$ and show that $g(\hat{x}) \geq 0$. This follows from two facts:

- $g(\hat{x}) \geq g(0)$, by the following argument. At every $x \in[0, \hat{x}], g$ has a non-negative derivative $g^{\prime}(x) \geq 0$ because $t_{+} \geq t_{-}$and because $f_{t}^{\prime}(x)$ is by MP increasing in $t \in T$. So $g$ is increasing, implying that $g(\hat{x}) \geq g(0)$.

- $g(0)=0$, by the following argument. For all $t \in T$ we have $f_{t}(0)=0$ because $(0,0) \in \mathbf{F}_{t}$ by MP. So $g(0)=f_{t_{+}}(0)-f_{t_{-}}(0)=0-0=0$, q.e.d.

Claim 4. For all $t \in T, \mathrm{y}_{\text {deter }}(t)=f_{t}\left(\mathrm{x}_{\text {deter }}(t)\right.$ ) (so I may henceforth write $f_{\bar{t}}(\bar{x})$ for $\mathrm{y}_{\text {deter }}(\bar{t})$, and $f_{\tilde{t}}(\tilde{x})$ for $\left.\mathrm{y}_{\text {deter }}(\tilde{t})\right)$.

Let $t \in T$. As $\mathbf{F}_{t}$ is topologically closed, it contains $\left(\mathrm{x}_{\text {deter }}(t), f_{t}\left(\mathrm{x}_{\text {deter }}(t)\right)\right)$ (by definition of $\left.f_{t}\right)$, i.e. $\left(\mathrm{x}_{\text {deter }}(t), f_{t}\left(\mathrm{x}_{\text {deter }}(t)\right)\right)$ is a feasible choice. So $\mathrm{y}_{\text {deter }}(t)$ cannot be larger than $f_{t}\left(\mathrm{x}_{\text {deter }}(t)\right)$ (otherwise $\left(\mathrm{x}_{\text {deter }}(t), \mathrm{y}_{\text {deter }}(t)\right)$ would be dispreferred to $\left(\mathrm{x}_{\text {deter }}(t), f_{t}\left(\mathrm{x}_{\text {deter }}(t)\right)\right)$ by punishment-aversion); it also cannot it be smaller than $f_{t}\left(\mathrm{x}_{\text {deter }}(t)\right)$ (otherwise $\left(\mathrm{x}_{\text {deter }}(t), \mathrm{y}_{\text {deter }}(t)\right)$ would be infeasible by definition of $f_{t}$ ), q.e.d.

Claim 5. $\bar{x}, \tilde{x} \in \mathbf{X}_{\tilde{t}} \subseteq \mathbf{X}_{\bar{t}}$ and $f_{\tilde{t}}(\tilde{x})-f_{\tilde{t}}(\bar{x}) \geq f_{\bar{t}}(\tilde{x})-f_{\bar{t}}(\bar{x}) \geq 0$.

By definition, $\tilde{x} \in \mathbf{X}_{\tilde{t}}$. Also $\bar{x} \in \mathbf{X}_{\tilde{t}}$, as $0 \leq \bar{x}<\tilde{x}$ and as $\mathbf{X}_{\tilde{t}}$ is an interval containing 0 (by Claim 1) and containing $\tilde{x}$. By Claim 1, $\mathbf{X}_{\tilde{t}} \subseteq \mathbf{X}_{\bar{t}}$. As for the inequalities, the second one holds because $\tilde{x}>\bar{x}$ and because $f_{\bar{t}}(x)$ is increasing in $x \in \mathbf{X}_{\bar{t}}$ by Claim 3. I now show the first inequality. For every $x \in \mathbf{X}_{\tilde{t}}$, since $f_{t}^{\prime}(x)$ is by MP increasing in $t \in T$, and since $\tilde{t}>\bar{t}$, we have $f_{\tilde{t}}^{\prime}(x) \geq f_{\bar{t}}^{\prime}(x)$, i.e. $\frac{d}{d x}\left(f_{\tilde{t}}(x)-f_{\bar{t}}(x)\right) \geq 0$. So $f_{\tilde{t}}(x)-f_{\bar{t}}(x)$ is a weakly increasing function of 
$x \in \mathbf{X}_{\tilde{t}}$. Hence, as $\tilde{x}>\bar{x}$, we have $f_{\tilde{t}}(\tilde{x})-f_{\tilde{t}}(\tilde{x}) \geq f_{\tilde{t}}(\bar{x})-f_{\bar{t}}(\bar{x})$, or by reordering, $f_{\tilde{t}}(\tilde{x})-f_{\tilde{t}}(\bar{x}) \geq f_{\bar{t}}(\tilde{x})-f_{\bar{t}}(\bar{x})$, q.e.d.

Claim 6. There is a counterexample to the condition TC.

I consider any $\delta>0$ and show that TC is violated when applied to the pairs $\left(\bar{x}, f_{\bar{t}}(\bar{x})\right)$ and $\left(\tilde{x}, f_{\bar{t}}(\tilde{x})+\delta\right)$ and to the 'punishment shift' $\epsilon:=f_{\tilde{t}}(\bar{x})-f_{\bar{t}}(\bar{x})$. To see that TC is applicable here, note three things.

- The first of the two pairs has lower punishment because $f_{\bar{t}}(\bar{x}) \leq f_{\bar{t}}(\tilde{x})<$ $f_{\bar{t}}(\tilde{x})+\delta$, where the first inequality follows from Claim 5 .

- The first pair is preferred to the second pair because $\left(\bar{x}, f_{\bar{t}}(\bar{x})\right) \succ_{\bar{t}}\left(\tilde{x}, f_{\bar{t}}(\tilde{x})\right) \succ_{\bar{t}}$ $\left(\tilde{x}, f_{\bar{t}}(\tilde{x})+\delta\right)$, where the second preference holds by punishment-aversion, and the first by the fact that $\left(\bar{x}, f_{\bar{t}}(\bar{x})\right)$ uniquely maximises $\succeq_{\bar{t}}$ within $\mathbf{F}_{\bar{t}}$.

- $\epsilon \geq 0$ because $\tilde{t}>\bar{t}$ and because $f_{t}(\bar{x})$ is by Claim 3 increasing in $t$. (To be precise, the condition TC is originally stated with a strict inequality ' $\epsilon>0$ ', but the condition trivially holds also if $\epsilon=0$.)

Now, by TC, $\left(\bar{x}, f_{\bar{t}}(\bar{x})+\epsilon\right) \succeq_{\bar{t}}\left(\tilde{x}, f_{\bar{t}}(\tilde{x})+\delta+\epsilon\right)$. So, as $\epsilon=f_{\tilde{t}}(\bar{x})-f_{\bar{t}}(\bar{x})$,

$$
\left(\bar{x}, f_{\tilde{t}}(\bar{x})\right) \succeq_{\bar{t}}\left(\tilde{x}, f_{\bar{t}}(\tilde{x})+f_{\tilde{t}}(\bar{x})-f_{\bar{t}}(\bar{x})+\delta\right) .
$$

In this, the right-hand side is weakly preferred to $\left(\tilde{x}, f_{\tilde{t}}(\tilde{x})+\delta\right)$, by punishmentaversion as by Claim $5 f_{\bar{t}}(\tilde{x})+f_{\tilde{t}}(\bar{x})-f_{\bar{t}}(\bar{x})+\delta \leq f_{\tilde{t}}(\tilde{x})+\delta$. So

$$
\left(\bar{x}, f_{\tilde{t}}(\bar{x})\right) \succeq_{\bar{t}}\left(\tilde{x}, f_{\tilde{t}}(\tilde{x})+\delta\right) .
$$

Since we have shown this for every $\delta>0$, and since by the continuity of $\succeq_{\bar{t}}$ any weak preference is preserved in the limit, we deduce that

$$
\left(\bar{x}, f_{\tilde{t}}(\bar{x})\right) \succeq_{\bar{t}} \lim _{\delta \downarrow 0}\left(\tilde{x}, f_{\tilde{t}}(\tilde{x})+\delta\right)=\left(\tilde{x}, f_{\tilde{t}}(\tilde{x})\right),
$$

a contradiction because $\left(\tilde{x}, f_{\tilde{t}}(\tilde{x})\right)$ uniquely maximises $\succeq_{\tilde{t}}$ within $\mathbf{F}_{\tilde{t}}$ and because $\left(\bar{x}, f_{\tilde{t}}(\bar{x})\right) \in \mathbf{F}_{\tilde{t}}$ (by definition of $f_{\tilde{t}}$ and the topological closedness of $\mathbf{F}_{\tilde{t}}$ ).

Proof of Theorem 2. Assume PM and CP. A close look at part (c) reveals that it follows from part (b) by taking the case that $P(t)=0$ for all $t \in T$ (no policy provokes); similarly, in (d), the claim referring to (c) follows from that referring to (b) by taking the mentioned special case. The proof of (a), (b) and the relevant part of (d) is done in several claims; (a) follows from Claims 2 and 9, (b) from Claim 10, and the relevant part of (d) from Claim 5.

Claim 1. For every policy $t \in T$, the feasible damage set $\mathbf{X}_{t} \subseteq \mathbf{R}_{+}$is a nonsingleton interval containing 0 , and the ratio $f_{t}(x) / x$ is an increasing function of $x \in \mathbf{X}_{t} \backslash\{0\}$.

Let $t \in T$. As $\mathbf{F}_{t}$ is by CP connected and contains $(0,0), \mathbf{X}_{t}$ is an interval containing 0 . $\mathbf{X}_{t}$ is non-singleton: otherwise $\mathbf{F}_{t}=\{(0,0)\}$, so that $(0,0)$ would be each type's best response to policy $t$, a trivial case excluded in PM. Now let $x, x^{\prime} \in \mathbf{X}_{t} \backslash\{0\}$ with $x<x^{\prime}$. The $x$ can be written as $\lambda x^{\prime}$ for some $0<\lambda<1$. 
So, as $f_{t}$ is by CP convex, $f(x) \leq \lambda f\left(x^{\prime}\right)+(1-\lambda) f(0)=\lambda f\left(x^{\prime}\right)$, whence $f(x) / x \leq \lambda f\left(x^{\prime}\right) / x=f\left(x^{\prime}\right) / x^{\prime}$, q.e.d.

Claim 2. For every policy $t \in T$, the function $f_{t}: \mathbf{X}_{t} \rightarrow \mathbf{R}_{+}$is increasing, continuous, and differentiable (from the right) at $x=0$ with $f_{t}^{\prime}(0) \geq 0$.

Let $t \in T$. The function $f_{t}$ is increasing on $\mathbf{X}_{t} \backslash\{0\}$ by Claim 1 , hence increasing on its full domain as $f_{t}(0)=0$ by $(0,0) \in \mathbf{F}_{t}$. As $x \downarrow 0$, the ratio $\frac{f(x)-f(0)}{x-0}=f(x) / x$ is decreasing (by Claim 1 ) and bounded below by 0 , hence has a limit $f^{\prime}(0)$ that is moreover $\geq 0$. It remains to show continuity. Since $f_{t}$ is convex, it is continuous on every open subinterval $I \subseteq \mathbf{X}_{t}$ (as the reader can easily check); so the only potential discontinuities arise at the boundaries of $\mathbf{X}_{t}$. Continuity at the left boundary 0 holds by differentiability. Now suppose $\mathbf{X}_{t}$ contains a right boundary, i.e. takes the form $\mathbf{X}_{t}=\left[0, x^{*}\right]$. and consider an increasing sequence $\left(x_{k}\right)_{k=1,2, \ldots}$ in $\mathbf{X}_{t}$ with $x_{k} \uparrow x^{*}$. As $f_{t}$ is increasing, the sequence $\left(f_{t}\left(x_{k}\right)\right)_{k=1,2, \ldots}$ is increasing and bounded (by $f_{t}\left(x^{*}\right)$ ), hence converges to a value $y$. So $\left(x_{k}, f_{t}\left(x_{k}\right)\right)_{k=1,2, \ldots}$ is a sequence in $\mathbf{F}_{t}$ that converges to $\left(a^{*}, y\right)$. So, as $\mathbf{F}_{t}$ is topologically closed, we have $\left(a^{*}, y\right) \in \mathbf{F}_{t}$, hence $f\left(a^{*}\right)=y$, q.e.d.

Claim 3. The function $d: \mathbf{R}_{+} \rightarrow \mathbf{R}$ is strictly increasing.

This follows easily from punishment-aversion, q.e.d.

Claim 4. For every policy $t \in T$, if $\mathbf{X}_{t}=\left[0, a^{*}\right)$ for some $a^{*} \equiv a_{t}^{*} \in \mathbf{R}_{+}$, then $f_{t}(x) \rightarrow \infty$ as $x \uparrow a^{*}$, and for every type $\theta$ there exists an $\epsilon \equiv \epsilon_{\theta} \in\left(0, a^{*}\right)$ such that $\left(x, f_{t}(x)\right) \prec_{t, \theta}(0,0)$ for all $x \in\left(a^{*}-\epsilon, a^{*}\right)$.

Let $t \in T$ and $\mathbf{X}_{t}=\left[0, a^{*}\right)$ with $a^{*} \in \mathbf{R}_{+}$.

First, if we had $f_{t}(x) \nrightarrow \infty$ as $x \uparrow a^{*}$, there would exist a sequence $\left(x_{k}\right)_{k=1,2, \ldots}$ in $\mathbf{X}_{t}$ with $x_{k} \rightarrow a^{*}$ such that the sequence $\left(x_{k}, f_{t}\left(x_{k}\right)\right)_{k=1,2, \ldots}$ is bounded in $\mathbf{R}_{+}^{2}$; now taking any convergent subsequence of it (there is one; see Th. A1.8 in Geoffrey Jehle and Philip Reny 2001), we would have a sequence in $\mathbf{F}_{t}$ that converges to some point $\left(a^{*}, y\right)$ outside $\mathbf{F}_{t}$, a contradiction since $\mathbf{F}_{t}$ is topologically closed.

Second, consider any type $\theta$. The claim is obvious if $\theta$ is a peaceful type. Now suppose $\theta \in \mathbf{R}$ and for a contradiction let no $\epsilon>0$ have the required property. Then there exists a sequence $\left(x_{k}\right)_{k=1,2, \ldots}$ in $\mathbf{X}_{t}$ such that $x_{k} \rightarrow a^{*}$ and $\left(x_{k}, f_{t}\left(x_{k}\right)\right) \succeq_{t, \theta}(0,0)$ for all $k=1,2, \ldots$ By $f_{t}\left(x_{k}\right) \rightarrow \infty$ and $x_{k} \rightarrow a^{*}$, we have $\alpha_{k}:=f_{t}\left(x_{k}\right) / x_{k} \rightarrow \infty$ as $k \rightarrow \infty$. In other words, the slope $\alpha_{k}$ of the straight line from $(0,0)$ to $\left(x_{k}, f_{t}\left(x_{k}\right)\right)$ tends to $\infty$ as $k \rightarrow \infty$. The point $\left(1 / \alpha_{k}, 1\right)$ is on this line; so, as $\left(x_{k}, f_{t}\left(x_{k}\right)\right) \succeq_{t, \theta}(0,0)$ and as preference $\succeq_{t, \theta}$ is convex, we have $\left(1 / \alpha_{k}, 1\right) \succeq_{t, \theta}(0,0)$ for all $k=1,2, \ldots$ It follows that $(0,1) \succeq_{t, \theta}(0,0)$, using that $\left(1 / \alpha_{k}, 1\right) \rightarrow(0,1)$ and that weak preferences are preserved in the limit because $\succeq_{t, \theta}$ is continuous (by the continuity of $u_{t, \theta}: \mathbf{R}_{+}^{2} \rightarrow \mathbf{R}$ ). This is a contradiction, because punishment-aversion requires that $(0,1) \prec_{t, \theta}(0,0)$, q.e.d.

Claim 5. Each type $\theta$ has a unique optimal response to each $t \in T$, denoted $\left(x_{t}^{\theta}, y_{t}^{\theta}\right)$; this optimum satisfies $y_{t}^{\theta}=f_{t}\left(x_{t}^{\theta}\right)$, and it is $(0,0)$ if $\theta$ is a peaceful type or a type in $\mathbf{R}$ with $\theta+P(t) \leq 0$.

Consider a $t \in T$. If a type has an optimum $(x, y) \in \mathbf{F}_{t}$ then it must 
be that $y=f_{t}(x)$, by punishment-aversion and since $\mathbf{F}_{t}$ contains $\left(x, f_{t}(x)\right)$ by topological closedness (see $\mathrm{CP}$ ).

All peaceful types $\theta$ and also all types $\theta \in \mathbf{R}$ with $\theta+P(t) \leq 0$ have peaceful (and punishment-averse) preferences $\succeq_{t, \theta}$, hence have the no-damageno-punishment pair $(0,0)$ as their unique optimal response to $t$.

It remains to consider a type $\theta \in \mathbf{R}$ with $\theta+P(t)>0$.

To show uniqueness, suppose for a contradiction that $\left(x_{1}, f_{t}\left(x_{1}\right)\right)$ and $\left(x_{2}, f_{t}\left(x_{2}\right)\right)$ are two distinct optimal responses to $t$. Consider any $x^{*}$ strictly between $x_{1}$ and $x_{2}$, say $x^{*}=\lambda x_{1}+(1-\lambda) x_{2}$ where $\lambda \in(0,1)$. The response $\left(x^{*}, f_{t}\left(x^{*}\right)\right)$ is of course feasible (i.e. in $\mathbf{F}_{t}$ ); I show that it is strictly preferred to $\left(x_{1}, f_{t}\left(x_{1}\right)\right)$, a contradiction. Let $y^{*}:=\lambda f_{t}\left(x_{1}\right)+(1-\lambda) f_{t}\left(x_{2}\right)$. As $f_{t}$ is convex, $y^{*} \geq$ $f_{t}\left(x^{*}\right)$, and so by punishment-aversion, $(*)\left(x^{*}, f_{t}\left(x^{*}\right)\right) \succeq_{t, \theta}\left(x^{*}, y^{*}\right)$. Moreover, by $\left(x^{*}, y^{*}\right)=\lambda\left(x_{1}, f_{t}\left(x_{1}\right)\right)+(1-\lambda)\left(x_{2}, f_{t}\left(x_{2}\right)\right)$, the strict convexity of $\succeq_{t, \theta}$ implies that $\left(x^{*}, y^{*}+\epsilon\right) \succeq_{t, \theta}\left(x_{1}, f_{t}\left(x_{1}\right)\right)$ for some $\epsilon>0$, and hence that $\left(x^{*}, y^{*}\right) \succ_{t, \theta}\left(x_{1}, f_{t}\left(x_{1}\right)\right)$ by punishment-aversion. The latter and $\left(^{*}\right)$ imply that $\left(x^{*}, f_{t}\left(x^{*}\right)\right) \succ_{t, \theta}\left(x_{1}, f_{t}\left(x_{1}\right)\right)$, the desired contradiction.

To show existence, I distinguish three cases.

Case 1: $\mathbf{X}_{t}$ is bounded and closed, i.e. of the compact form $\mathbf{X}_{t}=\left[0, x_{t}^{*}\right]$. As $f_{t}$ is continuous (by Claim 2) and $u_{t, \theta}$ is also continuous, $u_{t, \theta}\left(x, f_{t}(x)\right.$ ) is a continuous function of $x \in \mathbf{X}_{t}$, hence (as $\mathbf{X}_{t}$ is compact) admits a global maximum $\tilde{x} \in \mathbf{X}_{t}$ by Weierstrass' Theorem (Carl Simon and Lawrence Blume 1994, Th. 30.1). Now $\left(\tilde{x}, f_{t}(\tilde{x})\right)$ maximises $u_{t, \theta}(x, y)$ subject to $(x, y) \in \mathbf{F}_{t}$, as desired.

Case 2: $\mathbf{X}_{t}$ is bounded and open, i.e. of the form $\mathbf{X}_{t}=\left[0, x_{t}^{*}\right)$. By Claim 4, $\mathbf{X}_{t}$ has a subinterval of the form $\left[0, x_{t}^{*}-\epsilon\right]$ such that all feasible pairs $\left(x, f_{t}(x)\right)$ with $x \notin\left[0, x_{t}^{*}-\epsilon\right]$ are strictly dispreferred to some feasible pair $\left(x, f_{t}(x)\right)$ with $x \in\left[0, x_{t}^{*}-\epsilon\right]$ (in fact, strictly dispreferred to $(0,0)$ ). So it suffices to show that the function $u_{t, \theta}\left(x, f_{t}(x)\right)$ of $x$ admits a maximum on the compact subinterval $\left[0, x_{t}^{*}-\epsilon\right] \subseteq \mathbf{X}_{t}$. This can be done analogously to the proof in case 1 .

Case 3: $\mathbf{X}_{t}$ is unbounded, i.e. $\mathbf{X}_{t}=\mathbf{R}_{+}$. By an argument analogous to that in case 1 , on the compact subinterval $[0, \theta+P(t)]$ the function $u_{t, \theta}\left(x, f_{t}(x)\right)$ of $x$ admits a maximum $\tilde{x} \in[0, \theta+P(t)]$. I show that $\tilde{x}$ is a global maximum of $u_{t, \theta}\left(x, f_{t}(x)\right)$, which completes the proof as it establishes that $\left(\tilde{x}, f_{t}(\tilde{x})\right)$ is an optimal within $\mathbf{F}_{t}$. To do so, consider any $x \in \mathbf{R}_{+}$larger than $\theta+P(t)$. Recall that

$$
u_{t, \theta}\left(x, f_{t}(x)\right)=v(x-\theta-P(t))-d\left(f_{t}(x)\right) .
$$

So, as $v(x-\theta-P(t)) \leq v(0)$ (because $v$ peaks at 0$)$ and $d\left(f_{t}(x)\right) \geq d\left(f_{t}(\theta+P(t))\right)$ (because $f_{t}$ and $d$ are increasing functions by Claims 2 and 3 ),

$$
\left.u_{t, \theta}\left(x, f_{t}(x)\right) \leq v(0)-d\left(f_{t}(\theta+P(t))\right)=u_{t, \theta}(\theta+P(t)), f_{t}(\theta+P(t))\right),
$$

which is at most $u_{t, \theta}\left(\tilde{x}, f_{t}(\tilde{x})\right)$ by the restricted maximality property of $\tilde{x}$. This shows the global maximality property of $\tilde{x}$, q.e.d. 
Claim 6. The derivative $v^{\prime}: \mathbf{R} \rightarrow \mathbf{R}$ is strictly decreasing and $v^{\prime}(0)=0$.

This holds because $v$ is strictly concave and peaks at $x=0$.

Claim 7. The range $v^{\prime}(\mathbf{R})$ of $v^{\prime}$ is an interval containing 0 .

By the previous claim, $0=v^{\prime}(0) \in v^{\prime}(\mathbf{R})$. To prove that $v^{\prime}(\mathbf{R})$ is an interval (which if $v^{\prime}$ is continuous follows easily from the intermediate value theorem), it suffices to show that, for all $x_{1}, x_{2} \in v^{\prime}(\mathbf{R})$ with $x_{1}<x_{2}$ and all $s$ between $v^{\prime}\left(x_{1}\right)$ and $v^{\prime}\left(x_{2}\right)$ there exists an $\tilde{x} \in\left[x_{1}, x_{2}\right]$ such that $v^{\prime}(\tilde{x})=s$. Consider such $x_{1}, x_{2}, s$. By the previous claim, $v^{\prime}\left(x_{2}\right)<v^{\prime}\left(x_{1}\right)$, and hence $v^{\prime}\left(x_{2}\right)<s<v^{\prime}\left(x_{1}\right)$. I distinguish two cases, and write $k$ for the coefficient $\frac{v\left(x_{2}\right)-v\left(x_{1}\right)}{x_{2}-x_{1}}$.

Case 1: $s \geq k$. The (differentiable) function $g(x):=v(x)-\left[v\left(x_{1}\right)+s\left(x-x_{1}\right)\right]$ of $x \in \mathbf{R}$ satisfies

$$
g^{\prime}\left(x_{1}\right)=v^{\prime}\left(x_{1}\right)-s>0 .
$$

So, as $g\left(x_{1}\right)=0$, we have $g(\bar{x})>0$ for some $\bar{x} \in\left(x_{1}, x_{2}\right)$. Moreover,

$$
\begin{aligned}
g\left(x_{2}\right) & =v\left(x_{2}\right)-v\left(x_{1}\right)-s\left(x_{2}-x_{1}\right) \\
& \leq v\left(x_{2}\right)-v\left(x_{1}\right)-k\left(x_{2}-x_{1}\right)=0
\end{aligned}
$$

By $g(\bar{x})>0$ and $g\left(x_{2}\right) \leq 0$, and since $g$ is continuous, the intermediate value theorem implies the existence of some $\tilde{x} \in\left(\bar{x}, x_{2}\right]$ such that $g(\tilde{x})=0$. This means that $v(\tilde{x})=v\left(x_{1}\right)+s\left(\tilde{x}-x_{1}\right)$, i.e. that $\frac{v(\tilde{x})-v\left(x_{1}\right)}{\tilde{x}-x_{1}}=s$. Now we can apply the mean value theorem to the differentiable function $v$, which guarantees the existence of an $x \in\left[x_{1}, \tilde{x}\right]$ such that $v^{\prime}(x)=\frac{v(\tilde{x})-v\left(x_{1}\right)}{\tilde{x}-x_{1}}=s$, as desired.

Case 2: $s \leq k$. An argument analogous to that in Case 1 (based now on the function $g(x):=v(x)-\left[v\left(x_{2}\right)+s\left(x-x_{2}\right)\right]$ of $\left.x \in \mathbf{R}\right)$ guarantees again the existence of the desired $\tilde{x}$, q.e.d.

Claim 8. For all $t \in T$ and all $\theta \in \mathbf{R}$, (i) the function $U_{t, \theta}(x):=u_{t, \theta}\left(x, f_{t}(x)\right)$ of $x \in \mathbf{X}_{t}$ is differentiable (from the right) at $x=0$ with $U_{t, \theta}^{\prime}(0)=v^{\prime}(-\theta-$ $P(t))-d^{\prime}(0) f_{t}^{\prime}(0)$, and (ii) $x_{t}^{\theta}>0 \Leftrightarrow U_{t, \theta}^{\prime}(0)>0 \Leftrightarrow v^{\prime}(-\theta-P(t))>d^{\prime}(0) f_{t}^{\prime}(0)$.

Let $t \in T$ and $\theta \in \mathbf{R}$. Note that $\left.U_{t, \theta}(x)=v(x-\theta-P(t))-d\left(f_{t}(x)\right)\right)$ for all $x \in \mathbf{X}_{t}$. As $f_{t}$ is differentiable (from the right) at $x=0$ by Claim 1 , and as the functions $v$ and $d$ are differentiable, the chain rule implies that $U_{t, \theta}(x)$ is differentiable (from the right) at $x=0$ with derivative given by

$$
U_{t, \theta}^{\prime}(0)=v^{\prime}(-\theta-P(t))-d^{\prime}\left(f_{t}(0)\right) f_{t}^{\prime}(0)=v^{\prime}(-\theta-P(t))-d^{\prime}(0) f_{t}^{\prime}(0) .
$$

In (ii), the second equivalence is obvious by (i). Regarding the first equivalence, if $U_{t, \theta}^{\prime}(0)>0$ then, for some $x \in \mathbf{X}_{t}$, we have $U_{t, \theta}(0)<U_{t, \theta}(x)$, implying that $(0,0)=\left(0, f_{t}(0)\right) \prec_{t, \theta}\left(x, f_{t}(x)\right)$, hence that $(0,0)$ is not optimal, and so $x_{t, \theta}>0$. Conversely, suppose now that $x_{t}^{\theta}>0$. To show $U_{t, \theta}^{\prime}(0)>0$, I consider two cases.

Case 1: $d^{\prime}(0)=0$. Then $U^{\prime}(0)=v^{\prime}(-\theta-P(t))$, which is positive because $-\theta-P(t)<0$ (by $x_{t}^{\theta}>0$ and Claim 5) and because $v^{\prime}$ is positive on $(-\infty, 0)$ (by Claim 7). 
Case 2: $d^{\prime}(0)>0$. Since $\left(x_{t}^{\theta}, f_{t}\left(x_{t}^{\theta}\right)\right)=\left(x_{t}^{\theta}, y_{t}^{\theta}\right)$ is the unique optimal response to $t$ (by Claim 5), we have $\left(x_{t}^{\theta}, y_{t}^{\theta}\right) \succ_{t, \theta}\left(0, f_{t}(0)\right)=(0,0)$. So, as the preference $\succeq_{t, \theta}$ is continuity, there exists an $\epsilon>0$ such that $\left(x_{t}^{\theta}, y_{t}^{\theta}+\epsilon\right) \succ_{t, \theta}(0,0)$. Hence, as $\succeq_{t, \theta}$ is strictly convex, any strict convex combination of $\left(x_{t}^{\theta}, y_{t}^{\theta}+\epsilon\right)$ and $(0,0)$ is also strictly preferred to $(0,0)$. That is, $\left(\lambda x_{t}^{\theta}, \lambda\left(y_{t}^{\theta}+\epsilon\right)\right) \succ_{t, \theta}(0,0)$ for all $\lambda \in(0,1)$; or equivalently, $\left(x, x \frac{y_{t}^{\theta}+\epsilon}{x_{t}^{\theta}}\right) \succ_{t, \theta}(0,0)$ for all $x \in\left(0, x_{t}^{\theta}\right)$. So the function $V: \mathbf{X}_{t} \rightarrow \mathbf{R}$ defined by $V(x)=u_{t, \theta}\left(x, x \frac{y_{t}^{\theta}+\epsilon}{x_{t}^{\theta}}\right)$ satisfies $V(x) \geq V(0)$ whenever $x<x_{t}^{\theta}$. As $V$ is given by $V(x)=v(x-\theta-P(t))-d\left(x \frac{y_{t}^{\theta}+\epsilon}{x_{t}^{\theta}}\right)$ and as $v$ and $d$ are differentiable, the chain rule implies that $V$ is differentiable with derivative given by

$$
V^{\prime}(x)=v^{\prime}(x-\theta-P(t))-\frac{y_{t}^{\theta}+\epsilon}{x_{t}^{\theta}} d^{\prime}\left(x \frac{y_{t}^{\theta}+\epsilon}{x_{t}^{\theta}}\right) .
$$

As $V(x) \geq V(0)$ whenever $x<x_{t}^{\theta}$, we have $0 \leq V^{\prime}(0)$, i.e.

$$
0 \leq v^{\prime}(-\theta-P(t))-\frac{y_{t}^{\theta}+\epsilon}{x_{t}^{\theta}} d^{\prime}(0)<v^{\prime}(-\theta-P(t))-\frac{y_{t}^{\theta}}{x_{t}^{\theta}} d^{\prime}(0),
$$

where the latter inequality holds by $d^{\prime}(0)>0$. In the last expression,

$$
\frac{y_{t}^{\theta}}{x_{t}^{\theta}}=\frac{f_{t}\left(x_{t}^{\theta}\right)}{x_{t}^{\theta}} \geq \lim _{x \downarrow 0} \frac{f_{t}(x)}{x}=f_{t}^{\prime}(0)
$$

by Claims 1 and 2. This inequality and (9) imply that $0<v^{\prime}(-\theta-P(t))-$ $d^{\prime}(0) f_{t}^{\prime}(0)$, i.e. that $0<U^{\prime}(0)$, q.e.d.

Claim 9. For all $t \in T, d^{\prime}(0) f_{t}^{\prime}(0)$ has a unique inverse image $v^{\prime-1}\left(d^{\prime}(0) f_{t}^{\prime}(0)\right)$ under $v^{\prime}$ (denoted $\left.D(t)\right)$.

Let $t \in T$. Uniqueness of the inverse image holds because $v^{\prime}$ is a strictly decreasing (by Claim 6) and hence one-to-one. To show existence, I have to show that $d^{\prime}(0) f_{t}^{\prime}(0)$ is in the range $v^{\prime}(\mathbf{R})$. By PM, there exists a type $\theta \in \mathbf{R}$ for whom $(x, y)=(0,0)$ is not an optimal response to $t$; so the optimum $\left(x_{t}^{\theta}, f_{t}\left(x_{t}^{\theta}\right)\right)$ satisfies $x_{t}^{\theta}>0$. Hence, by Claim 8, $v^{\prime}(-\theta-P(t))>d^{\prime}(0) f_{t}^{\prime}(0)$. As we also have $d^{\prime}(0) f_{t}^{\prime}(0) \geq 0$ (by Claims 1 and 2), $d^{\prime}(0) f_{t}^{\prime}(0) \in\left[0, v^{\prime}(-\theta-P(t))\right]=$ $\left[v^{\prime}(0), v^{\prime}(-\theta-P(t))\right]$. So, as $\left[v^{\prime}(0), v^{\prime}(-\theta-P(t))\right] \subseteq v^{\prime}(\mathbf{R})$ (because $v^{\prime}(\mathbf{R})$ is an interval by Claim 7$), d^{\prime}(0) f_{t}^{\prime}(0) \in v^{\prime}(\mathbf{R})$, q.e.d.

Claim 10. For all policies $t \in T$, a type $\theta \in \mathbf{R}$ has $x_{t}^{\theta}>0$ (i.e. is a terrorist) if and only $\theta>-(P(t)+D(t))$.

Let $t \in T$ and $\theta \in \mathbf{R}$. We have $x_{t}^{\theta}>0$ if and only if $v^{\prime}(-\theta-P(t))>$ $d^{\prime}(0) f_{t}^{\prime}(0)$. The function $v^{\prime}: \mathbf{R} \rightarrow \mathbf{R}$ is by Claim 6 strictly decreasing, hence is invertible with strictly decreasing inverse function $v^{\prime-1}$, whose domain $v(\mathbf{R})$ contains both sides of the last inequality by Claim 9. So, applying $v^{\prime-1}$ on both sides of the inequality yields the equivalent inequality $-\theta-P(t)<v^{\prime-1}\left(d^{\prime}(0) f_{t}^{\prime}(0)\right)$ $(=D(t))$, which in turn is equivalent to $\theta>-(P(t)+D(t))$. 
Derivations for the example after Theorem 2. All functions in the example satisfy the required differentiability or convexity conditions. At all $x \leq 0$ we have $v^{\prime}(x)=\left(-(-x)^{a}\right)^{\prime}=a(-x)^{a-1}$. So the inverse $v^{\prime}: \mathbf{R} \rightarrow \mathbf{R}$ is at all $z \geq 0$ given by $v^{\prime-1}(z)=-(z / a)^{1 /(a-1)}$. Hence $D(t)=v^{\prime-1}\left(d^{\prime}(0) f_{t}^{\prime}(0)\right)=v^{\prime-1}(b t)=$ $-(b t / a)^{1 /(a-1)}$. To minimise $P(t)+D(t)$, note that at all $t>0$ this function is differentiable with derivative

$$
\begin{aligned}
\frac{d}{d t}[P(t)+D(t)] & =\frac{d}{d t}\left[(t-\bar{t}) p-\left(\frac{t b}{a}\right)^{\frac{1}{a-1}}\right] \\
& =p-\frac{(b / a)^{\frac{1}{a-1}}}{a-1} t^{\frac{1}{a-1}-1}=p-\frac{(b / a)^{\frac{1}{a-1}}}{a-1} t^{\frac{2-a}{a-1}},
\end{aligned}
$$

which is zero if and only if

$$
t=\left[\frac{p(a-1)}{(b / a)^{1 /(a-1)}}\right]^{\frac{a-1}{2-a}}=\left[\frac{(b / a)^{1 /(a-1)}}{p(a-1)}\right]^{\frac{a-1}{a-2}}=\frac{(b / a)^{1 /(a-2)}}{(p(a-1))^{(a-1) /(a-2)}} .
$$

This only stationary point of $P(t)+D(t)$ is indeed a (global) minimum, since the above expression for $\frac{d}{d t}[P(t)+D(t)]$ is strictly increasing in $t>0$ (using that $a>2)$. 